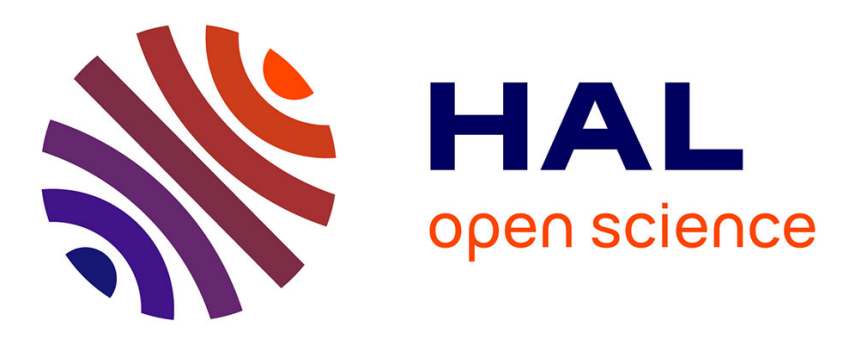

\title{
Localization of Vibratory Energy of Main Linear/Nonlinear Structural Systems by Nonlinear Energy Sink
}

Claude-Henri Lamarque, Alireza Ture Savadkoohi

\section{- To cite this version:}

Claude-Henri Lamarque, Alireza Ture Savadkoohi. Localization of Vibratory Energy of Main Linear/Nonlinear Structural Systems by Nonlinear Energy Sink. Belhaq M. Structural Nonlinear Dynamics and Diagnosis, 168, Srpinger, 2015, Springer Proceedings in Physics, 978-3-319-19851-4. 10.1007/978-3-319-19851-4_11. hal-01638173

\section{HAL Id: hal-01638173 https://hal.science/hal-01638173}

Submitted on 19 Nov 2017

HAL is a multi-disciplinary open access archive for the deposit and dissemination of scientific research documents, whether they are published or not. The documents may come from teaching and research institutions in France or abroad, or from public or private research centers.
L'archive ouverte pluridisciplinaire HAL, est destinée au dépôt et à la diffusion de documents scientifiques de niveau recherche, publiés ou non, émanant des établissements d'enseignement et de recherche français ou étrangers, des laboratoires publics ou privés. 


\title{
Localization of Vibratory Energy of Main Linear/Nonlinear Structural Systems by Nonlinear Energy Sink
}

\author{
C.-H. Lamarque and A. Ture Savadkoohi
}

\begin{abstract}
Two systems are considered: the system I is composed of a main linear structure which is coupled to a nonsmooth nonlinear energy sink. Here, effects of the gravity forces are not neglected. The system II consists of a main structure with a set of parallel Saint-Venant elements that is attached to a nonlinear energy sink with general odd nonlinear potential function. Time multi-scale energy exchanges between two oscillators is detected; in detail: the invariant manifold of the system at fast time scale is traced while detected equilibrium and singular points at slow time scale give us envision about system behavior(s) at pseudo-steady-state regime(s). All of detected behaviors provide us design tools for tuning necessary parameters of nonlinear energy sink for the localization of vibratory energy of main structural systems.
\end{abstract}

\section{Introduction}

It has been proved that pumping the essential part of vibratory energy of main structures is possible by endowing nonlinear properties of coupled oscillators [1, 2]. Later on this phenomenon was used for transferring the energy of main systems to secondary oscillators with very small mass compared to the main one's and with essential cubic geometrical nonlinearity. The phenomenon is named as "energy pumping" and the light and nonlinear oscillator is called as nonlinear energy sink (NES) [3-35]. Efficiency of the NES in localization of vibratory energy and passive control of systems has been proved experimentally as well [36-43]. However in most of above mentioned studies, the nonlinearity of the NES is cubic and the main structural system is supposed to be linear or to present smooth nonlinearity [16, 26, 27]. Some research works have been carried out to consider other types of nonlinearities for

C.-H. Lamarque · A.Ture Savadkoohi

ENTPE, Université de Lyon, LGCB and LTDS UMR CNRS 5513,

Rue Maurice Audin, 69518 Vaulx En Velin, France

e-mail: lamarque@entpe.fr

\footnotetext{
A.Ture Savadkoohi

e-mail: alireza.turesavadkoohi@entpe.fr
} 
the geometrical potential of the NES and their efficiency in passive control of main systems, e.g. vibro-impact and non-polynomial nonlinearities, non-smooth potential function with constant or time-dependent mass [44-50]. There have been some research works that consider nonlinear main structural system to be controlled by cubic or non-smooth NES; in detail: a main oscillator with piece-wise linear and also Dahl-type behavior and a coupled nonsmooth NES [51, 52]; the main system with hysteresis behavior of Bouc-Wen type and a NES with general nonlinear potential function [53]; the main structure with single or several Saint-Venant elements [54] in parallel and a NES with cubic or general potential function $[55,56]$. The current paper is a summary of our two previous research works which deals with the localization of vibratory energy of: (i) vertical main structural systems by a nonsmooth NES [48] and (ii) main structural systems with a set of parallel Saint-Venant elements by a NES with general potential function [56]. Organization of the chapter is as it follows: summary of the general methodology to deal with multiple scale dynamics of a main oscillator and a coupled NES is given in Sect. 2. Energy exchanges between a vertical linear system and a coupled nonsmooth NES is presented in Sects. 3 and 4 deals with studying of the dynamics of a main structural system including a set of parallel Saint-Venant elements and a coupled NES with a general nonlinear potential function. Finally conclusions are collected in Sect. 5.

\section{General Methodology to Deal with Two Coupled Oscillators: A Main System + NES}

In order to study multiple energy exchanges between a main oscillator and a coupled NES, we implement following steps [53]:

- re-scaling the system.

- transferring the system to the center of mass and relative displacement.

- applying complex variables to the system and using Galerkin technique by keeping first harmonics (and constant terms).

- embedding the time to different scales (fast and slow time scales) and detecting invariant manifold at the fast time scale and equilibrium points and fold singularities of the reduced order form of the system at the slow time scale.

\section{Localization of Vibratory Energy of Vertical Main Linear Structural Systems by Coupled Non-smooth NES}

Here we would like to analyze time multi-scale energy exchanges between a linear system and a nonsmooth NES. Gravity loads are not neglected. Let us consider the academic model of a system which is subjected to vertical excitations as is depicted 
Fig. 1 The academic model of the system under gravity $(g)$ loads and external force $\Gamma \sin (\Omega t) . m=\varepsilon M$,

$0<\varepsilon \ll 1$; masses oscillate vertically

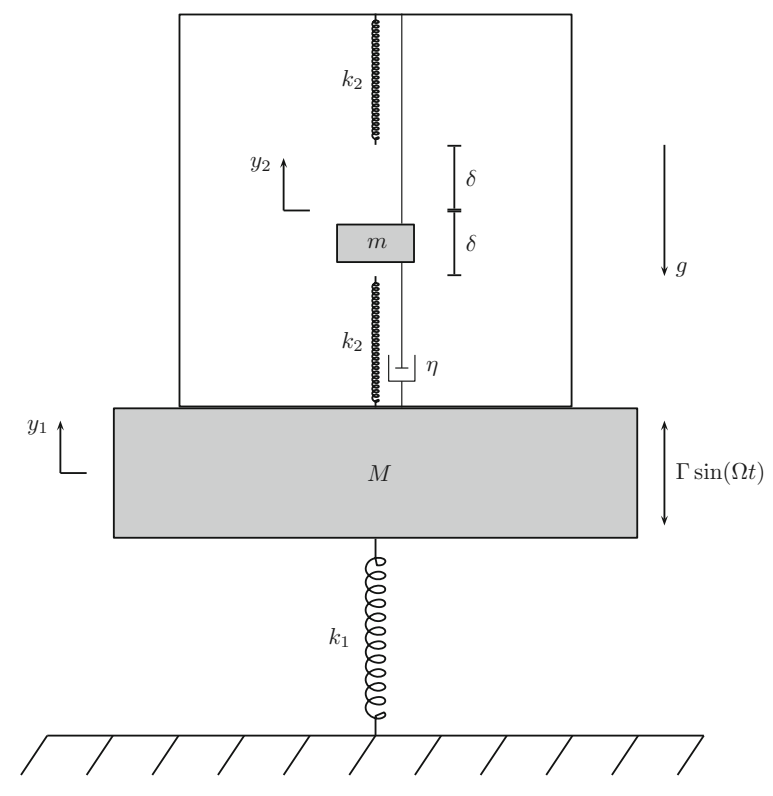

in Fig. 1. The system is composed of a linear main structure $(M)$ which is coupled to a non-smooth NES $(m)$ system. Governing system equations can be summarized as:

$$
\left\{\begin{array}{l}
M \ddot{y}_{1}+k_{1} y_{1}+F\left(y_{1}-y_{2}\right)+\eta\left(\dot{y}_{1}-\dot{y}_{2}\right)+M g=\Gamma \sin (\Omega t) \\
m \ddot{y}_{2}+F\left(y_{2}-y_{1}\right)+\eta\left(\dot{y}_{2}-\dot{y}_{1}\right)+m g=0
\end{array}\right.
$$

where $\eta$ is the continues linear damping of the NES and $F$ is the non-smooth potential function of the NES which is defined as follows:

$$
F(z)=-\frac{\partial V(z)}{\partial z}=-F(-z)=\left\{\begin{array}{lc}
0 & \text { if }-\delta \leq z \leq \delta \\
k_{2}(z-\delta) & \text { if } \quad z \geq \delta \\
k_{2}(z+\delta) & \text { if } \quad z \leq-\delta
\end{array}\right.
$$

If we suppose that $T=t \sqrt{\frac{k_{1}}{M}}=t \vartheta$, then the system (1) in the $T$ domain reads:

$$
\left\{\begin{array}{l}
\ddot{y}_{1}+y_{1}+\frac{1}{k_{1}} F\left(y_{1}-y_{2}\right)+\frac{\eta}{\sqrt{k_{1} M}}\left(\dot{y}_{1}-\dot{y}_{2}\right)+\frac{M g}{k_{1}}=\frac{\Gamma \sin \left(\frac{\Omega}{\vartheta} T\right)}{k_{1}} \\
\varepsilon \ddot{y}_{2}+\frac{1}{k_{1}} F\left(y_{2}-y_{1}\right)+\frac{\eta}{\sqrt{k_{1} M}}\left(\dot{y}_{2}-\dot{y}_{1}\right)+\frac{m g}{k_{1}}=0
\end{array}\right.
$$


We introduce the following variables: $\varepsilon=\frac{m}{M}, \gamma=\frac{M g}{k_{1}}, \varepsilon \lambda=\frac{\eta}{\sqrt{k_{1} M}}, \frac{1}{k_{1}} F(z)=$ $\varepsilon \tilde{F}(z), k=\frac{1}{\varepsilon} \frac{k_{2}}{k_{1}}, \omega=\frac{\Omega}{\vartheta}$ and $\frac{\Gamma}{k_{1}}=\varepsilon f_{0}$. So, scaled potential of the NES reads:

$$
\tilde{F}(z)= \begin{cases}0 & \text { if }-\delta \leq z \leq \delta \\ k(z-\delta) & \text { if } \quad z \geq \delta \\ k(z+\delta) & \text { if } \quad z \leq-\delta\end{cases}
$$

Following system can be derived:

$$
\left\{\begin{array}{l}
\ddot{y}_{1}+y_{1}+\varepsilon \tilde{F}\left(y_{1}-y_{2}\right)+\varepsilon \lambda\left(\dot{y}_{1}-\dot{y}_{2}\right)+\gamma=\varepsilon f_{0} \sin (\omega T) \\
\varepsilon \ddot{y}_{2}+\varepsilon \tilde{F}\left(y_{2}-y_{1}\right)+\varepsilon \lambda\left(\dot{y}_{2}-\dot{y}_{1}\right)+\varepsilon \gamma=0
\end{array}\right.
$$

We are interested to study forced vibration but also the transient behavior occurring before reaching the steady-state regime. The frequency of the main system in (5) is equal to " 1 " and we would like to analyze system behavior in the vicinity of 1:1 resonance. We will suppose that $\omega=1+\sigma \varepsilon$ and since $\varepsilon$ is a small, it means that we will pinpoint system behavior around 1:1 resonance. Let us transfer the system to the following coordinates:

$$
\left\{\begin{array}{l}
v=y_{1}+\varepsilon y_{2} \\
w=y_{1}-y_{2}
\end{array}\right.
$$

In the second equation of the system (5), the parameter $\varepsilon$ has been kept intentionally in order to show coupling terms between two equations of the system (5) and physical orders of respective equations. Especially $v$ is associated to the center of two masses $(1, \varepsilon)$. If we divide the second equation of the system (5) by $\varepsilon$, then we will have a system of two masses at the same order $(1,1)$, leading to $v=y_{1}+y_{2}$ and $w=y_{1}-y_{2}$ which do not have appropriate physical meanings for the system under consideration. The consequence should be to keep nonlinear terms in both resulting equations for $v$ and $w$.

By adding and subtraction two equations of the system (5) we will have:

$$
\left\{\begin{array}{l}
\ddot{v}+\frac{1}{1+\varepsilon}(v+\varepsilon w)+\gamma(1+\varepsilon)=\varepsilon f_{0} \sin (\omega T) \\
\ddot{w}+\frac{1}{1+\varepsilon}(v+\varepsilon w)+(1+\varepsilon) \tilde{F}(w)+(1+\varepsilon) \lambda \dot{w}=\varepsilon f_{0} \sin (\omega T)
\end{array}\right.
$$

We introduce a modified form of Manevitch's complex variables [57] to the system (7): 


$$
\left\{\begin{array}{l}
B_{1}+\varphi_{1} e^{i \omega T}=\dot{v}+i \omega v \\
B_{2}+\varphi_{2} e^{i \omega T}=\dot{w}+i \omega w
\end{array}\right.
$$

with $B_{1}=i b_{1}, B_{2}=i b_{2}$ and $i^{2}=-1 . B_{1}$ and $B_{2}$ are constant terms taking into account the fact that the dynamical system (5) (including $\gamma$ ) is not written around the $\left(y_{1}, y_{2}, \dot{y}_{1}, \dot{y}_{2}\right)=(0,0,0,0)$. So, we have to include constant terms to take into account nonlinear terms in averaging. It corresponds to taking into account constant terms of Fourier series together with first harmonic terms. We can present the function $\tilde{F}(w)$ in the form of Fourier series:

$$
\tilde{F}(w)=\tilde{F}\left(\frac{b_{2}}{\omega}-\frac{i}{2 \omega}\left(\varphi_{2} e^{i \omega T}-\varphi_{2}^{*} e^{-i \omega T}\right)\right)=\sum_{j=-\infty}^{+\infty} f_{j}\left(b_{2}, \varphi_{2}, \varphi_{2}^{*}\right) e^{i \omega j T}
$$

where the * represents the complex conjugate of the function under consideration. We implement the Galerkin method using a truncated Fourier series (constant and first harmonic) and then we endow multiple time scales approach to investigate the evolution of the Fourier-coefficients. The constant and first harmonic of the (7) by considering variables of (8) and $j=0,1$ in (9) read as:

$$
\begin{gathered}
\left\{\begin{array}{l}
\frac{1}{1+\varepsilon}\left(\frac{b_{1}}{\omega}+\varepsilon \frac{b_{2}}{\omega}\right)+(1+\varepsilon) \gamma=0 \\
\frac{1}{1+\varepsilon}\left(\frac{b_{1}}{\omega}+\varepsilon \frac{b_{2}}{\omega}\right)+(1+\varepsilon) f_{z}\left(b_{2}, \varphi_{2}, \varphi_{2}^{*}\right)=0
\end{array}\right. \\
\left\{\begin{array}{l}
\dot{\varphi}_{1}=-\frac{i}{2} \varepsilon f_{0}+\frac{i}{2 \omega(1+\varepsilon)}\left(\varphi_{1}+\varepsilon \varphi_{2}\right)-\frac{i}{2} \omega \varphi_{1} \\
\dot{\varphi}_{2}=-\frac{i}{2} \varepsilon f_{0}+\frac{i}{2 \omega(1+\varepsilon)}\left(\varphi_{1}+\varepsilon \varphi_{2}\right)-\frac{\lambda(1+\varepsilon)}{2} \varphi_{2}-\frac{i}{2} \omega \varphi_{2}-(1+\varepsilon) f_{f}\left(b_{2}, \varphi_{2}, \varphi_{2}^{*}\right)
\end{array}\right.
\end{gathered}
$$

where $f_{z}\left(b_{2}, \varphi_{2}, \varphi_{2}^{*}\right)$ and $f_{f}\left(b_{2}, \varphi_{2}, \varphi_{2}^{*}\right)$ are zero and first Fourier coefficients which can be evaluated as:

$$
\begin{gathered}
f_{z}\left(b_{2}, \varphi_{2}, \varphi_{2}^{*}\right)=\frac{\omega}{2 \pi} \int_{0}^{\frac{2 \pi}{\omega}} \tilde{F}\left(\frac{b_{2}}{\omega}-\frac{i}{2 \omega}\left(\varphi_{2} e^{i \omega T}-\varphi_{2}^{*} e^{-i \omega T}\right)\right) d T \\
f_{f}\left(b_{2}, \varphi_{2}, \varphi_{2}^{*}\right)=\frac{\omega}{2 \pi} \int_{0}^{\frac{2 \pi}{\omega}} \tilde{F}\left(\frac{b_{2}}{\omega}-\frac{i}{2 \omega}\left(\varphi_{2} e^{i \omega T}-\varphi_{2}^{*} e^{-i \omega T}\right)\right) e^{-i \omega T} d T
\end{gathered}
$$

It can be proved that [48]:

$$
f_{f}\left(b_{2}, \varphi_{2}, \varphi_{2}^{*}\right)=-\frac{i \varphi_{2}}{2} G_{f}\left(\left|\varphi_{2}\right|^{2}\right)
$$


where for any variable $\chi \geq 0$

$$
G_{f}(\chi)=\left\{\begin{array}{l}
0 \quad \text { if } \frac{b_{2}}{\omega}+\frac{\sqrt{\chi}}{\omega}<\delta \\
\frac{k}{2 \pi \omega}\left(\pi+\frac{2 b_{2}}{\chi} \sqrt{\chi-\left(b_{2}-\delta \omega\right)^{2}}-\frac{2 \delta \omega}{\chi} \sqrt{\chi-\left(b_{2}-\delta \omega\right)^{2}}-\right. \\
\frac{2 b_{2}}{\chi} \sqrt{\chi-\left(b_{2}+\delta \omega\right)^{2}}-\frac{2 \delta \omega}{\chi} \sqrt{\chi-\left(b_{2}+\delta \omega\right)^{2}}+ \\
\left.2 \arccos \left(\frac{b_{2}+\delta \omega}{\sqrt{\chi}}\right)+2 \arcsin \left(\frac{b_{2}-\delta \omega}{\sqrt{\chi}}\right)\right) \quad \text { if } \frac{b_{2}}{\omega}+\frac{\sqrt{\chi}}{\omega} \geq \delta
\end{array}\right.
$$

and

$$
f_{z}(\chi)=\left\{\begin{array}{l}
0 \quad \text { if } \frac{b_{2}}{\omega}+\frac{\sqrt{\chi}}{\omega}<\delta \\
\frac{k}{\pi \omega}\left(b_{2} \pi+\sqrt{\left(b_{2}+\sqrt{\chi}-\delta \omega\right)(-b+\sqrt{\chi}+\delta \omega)}-\right. \\
\sqrt{\left(-b_{2}+\sqrt{\chi}-\delta \omega\right)\left(b_{2}+\sqrt{\chi}+\delta \omega\right)}+ \\
\left.\left(b_{2}-\delta \omega\right) \arcsin \left(\frac{b_{2}-\delta \omega}{\sqrt{\chi}}\right)+\left(b_{2}+\delta \omega\right) \arcsin \left(\frac{b_{2}+\delta \omega}{\sqrt{\chi}}\right)\right) \\
\quad \text { if } \frac{b_{2}}{\omega}+\frac{\sqrt{\chi}}{\omega} \geq \delta
\end{array}\right.
$$

To deal with the systems (10) and (11), an asymptotic approach [58] by introducing slow times $\tau_{1}, \tau_{2}, \ldots$ with the fast time $\tau_{0}$ can be implemented as follows:

$$
T=\tau_{0}, \quad \tau_{1}=\varepsilon \tau_{0}, \ldots,
$$

so,

$$
\frac{d}{d T}=\frac{\partial}{\partial \tau_{0}}+\varepsilon \frac{\partial}{\partial \tau_{1}}+\cdots
$$

In the next sections we will try to have finer envision into systems (10) and (11) at different orders of $\varepsilon$ in order to grasp the system behavior during different scales of time. 


\subsection{Truncated Fourier Series: Constant Term}

The general form of the system (10) show that $\gamma=f_{z}\left(N_{2}^{2}\right)$. During the $\varepsilon^{0}$ order, it leads to:

$$
b_{1}=-\gamma
$$

while during the $\varepsilon^{1}$ order we have:

$$
b_{2}=-\gamma(2+\sigma)
$$

\subsection{Truncated Fourier Series: First Harmonic}

\subsection{1 $\varepsilon^{0}$ Order}

We assume that the system is around 1:1 resonance $(\omega=1+\sigma \varepsilon)$. Equation (11) at the $\varepsilon^{0}$ order yield to:

$$
\begin{gathered}
\frac{\partial \varphi_{1}}{\partial \tau_{0}}=0 \Rightarrow \varphi_{1}=\varphi_{1}\left(\tau_{1}\right) \\
\frac{\partial \varphi_{2}}{\partial \tau_{0}}+\frac{i\left(1-G_{f}\left(\left|\varphi_{2}\right|^{2}\right)\right)+\lambda}{2} \varphi_{2}=\frac{i}{2} \varphi_{1}
\end{gathered}
$$

so, fixed points of the system $\left(\Phi\left(\tau_{1}\right)\right)$ can be evaluated by following equation:

$$
\frac{i\left(1-G_{f}\left(|\Phi|^{2}\right)\right)+\lambda}{2} \Phi=\frac{i}{2} \varphi_{1}
$$

Let us assume that $\varphi_{1}=N_{1} e^{i \delta_{1}}$ and $\Phi=N_{2} e^{i \delta_{2}}$, so (23) can be re-written as the following form:

$$
N_{2}-i \lambda N_{2}-G_{f}\left(N_{2}^{2}\right) N_{2}=N_{1} e^{i\left(\delta_{1}-\delta_{2}\right)}
$$

which leads us to follow invariant manifold of the system during $\tau_{0}$ time scale $\left(\tau_{0^{-}}\right.$ invariant):

$$
N_{1}=N_{2} \sqrt{\lambda^{2}+\left(1-G_{f}\left(N_{2}^{2}\right)\right)^{2}}
$$

A typical invariant manifold for given system parameters is illustrated in Fig. 2. It has been proved that stability borders of the $\tau_{0}$-invariant is defined as it follows [48]:

$$
\lambda^{2}+\left(1-G_{f}\left(N_{2}^{2}\right)\right)\left(1-H\left(N_{2}^{2}\right) N_{2}-G_{f}\left(N_{2}^{2}\right)\right)>0
$$


Fig. $2 \tau_{0}$-invariant manifold of the system and its stable and unstable zones

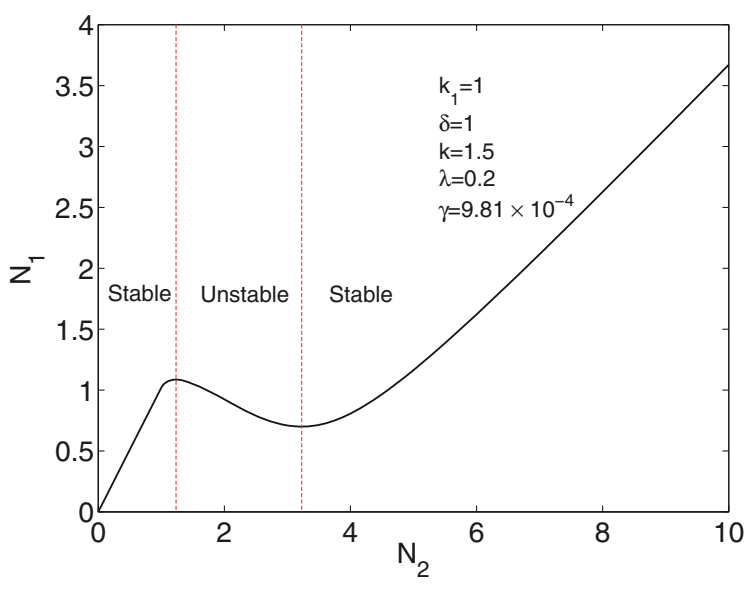

Stable and unstable zones of the invariant manifold are depicted in Fig. 2. In fact when the system arrives to the vicinity of the unstable zone, it tries to reach another stable zone via a bifurcation. This will lead to the energy pumping phenomenon.

\subsection{2 $\varepsilon^{1}$ Order}

At the order of $\varepsilon^{1}$ the first equation of the system (11) reads as:

$$
\frac{\partial \varphi_{1}}{\partial \tau_{1}}=-\frac{i}{2} f_{0}+\frac{i}{2}\left(\Phi-\varphi_{1}-\sigma \varphi_{1}\right)-\frac{i}{2} \sigma \varphi_{1}
$$

Let us try to enlighten the behavior of the system at the $\tau_{1}$ time scale "around" the invariant manifold at the time scale $\tau_{0}$. By considering (23), following system can be derived:

$$
\begin{aligned}
& \frac{\partial}{\partial \tau_{1}}\left(\Phi-i \lambda \Phi-\Phi G_{f}\left(|\Phi|^{2}\right)\right) \\
& =\frac{i}{2}\left(-f_{0}-2 \sigma\left(\Phi-i \lambda \Phi-G_{f}\left(|\Phi|^{2}\right) \Phi\right)+i \lambda \Phi+G_{f}\left(|\Phi|^{2}\right) \Phi\right)
\end{aligned}
$$

and if we suppose that $\Phi=N_{2}\left(\tau_{1}\right) e^{i \delta_{2}\left(\tau_{1}\right)}$, following compact for of equations can be obtained:

$$
\begin{gathered}
\frac{\partial N_{2}}{\partial \tau_{1}}=\frac{f_{1}\left(N_{2}, \delta_{2}\right)}{g\left(N_{2}\right)} \\
\frac{\partial \delta_{2}}{\partial \tau_{1}}=\frac{f_{2}\left(N_{2}, \delta_{2}\right)}{g\left(N_{2}\right)}
\end{gathered}
$$


where,

$$
\begin{gathered}
f_{1}\left(N_{2}, \delta_{2}\right)=f_{0} \sin \left(\delta_{2}\right)\left(G_{f}\left(N_{2}^{2}\right)-1\right)-\lambda N_{2}+\lambda f_{0} \cos \left(\delta_{2}\right) \\
f_{2}\left(N_{2}, \delta_{2}\right)=-\frac{1-G_{f}\left(N_{2}^{2}\right)-2 N_{2}^{2} G_{f}^{\prime}\left(N_{2}^{2}\right)}{N_{2}} f_{0} \cos \left(\delta_{2}\right)-\frac{\lambda}{N_{2}} f_{0} \sin \left(\delta_{2}\right) \\
-\lambda^{2}(1+2 \sigma)+\left(1-G_{f}\left(N_{2}^{2}\right)-2 N_{2}^{2} G_{f}^{\prime}\left(N_{2}^{2}\right)\right)\left(-2 \sigma+2 \sigma G_{f}\left(N_{2}^{2}\right)+G_{f}\left(N_{2}^{2}\right)\right) \\
g\left(N_{2}\right)=2\left(1+\lambda^{2}-2 G_{f}\left(N_{2}^{2}\right)-2 N_{2}^{2} G_{f}^{\prime}\left(N_{2}^{2}\right)+G_{f}^{2}\left(N_{2}^{2}\right)+2 N_{2}^{2} G_{f}\left(N_{2}^{2}\right) G_{f}^{\prime}\left(N_{2}^{2}\right)\right)
\end{gathered}
$$

The relation $g\left(N_{2}\right)=0$ provides two values for $N_{2}$, namely $N_{21}$ and $N_{22}$. They are called as fold lines of the system. We will use these equations later on for detailed bifurcation analysis of the system.

For detecting the invariant manifold of the system at the $\tau_{1}$ time scale ( $\tau_{1}$-invariant), (28) can be re-written as $\left(\frac{\partial \varphi_{1}}{\partial \tau_{1}}=0\right)$ :

$$
\frac{i}{2}\left(-f_{0}-2 \sigma\left(\Phi-i \lambda \Phi-G_{f}\left(|\Phi|^{2}\right) \Phi\right)+i \lambda \Phi+G_{f}\left(|\Phi|^{2}\right) \Phi\right)=0
$$

or

$$
f_{0}=N_{2} \sqrt{\lambda^{2}(1+2 \sigma)^{2}+\left((1+2 \sigma) G_{f}\left(N_{2}^{2}\right)-2 \sigma\right)^{2}}
$$

A typical invariant manifold of the system at the time scale $\tau_{1}$ is depicted in Fig. 3 . Intersections of this invariant manifold (for a given $f_{0}$ ) with the $\tau_{0}$-invariant are positions of fixed points.

Fig. $3 \tau_{1}$-invariant manifold of the system

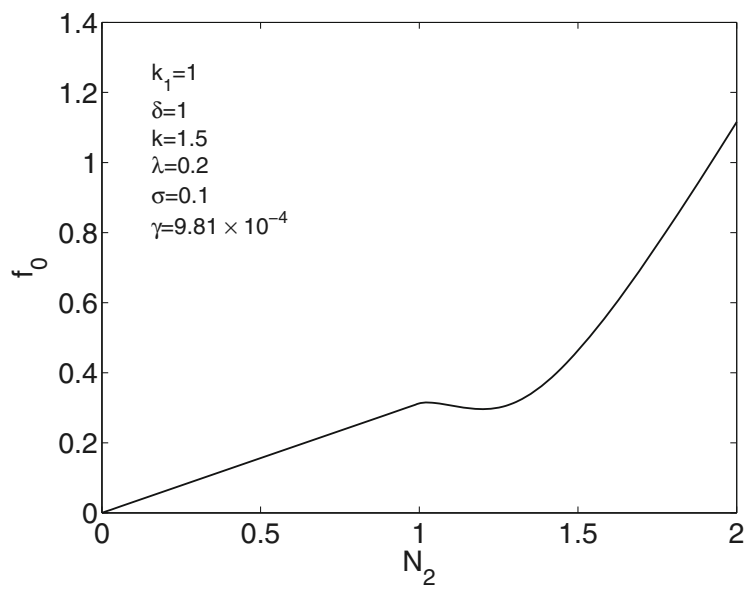




\subsection{Analytical Results Versus Numerical Integrations}

Since the overall system is under gravity loading, the initial equilibrium state of each mass $\left(y_{10}\right.$ and $\left.y_{20}\right)$ should be evaluated. Let us consider different hypothesis as the equilibrium state for the system (1):

- $-\delta<y_{2}-y_{1}<+\delta$ so $F\left(y_{2}-y_{1}\right)=0$

This assumption leads us to following system:

$$
\left\{\begin{array}{l}
k_{1} y_{1}+M g=0 \\
m g=0
\end{array}\right.
$$

which is an impossible equality. So, this assumption is not valid at the equilibrium state of the overall system.

- $y_{2}-y_{1}<-\delta$ so $F\left(y_{2}-y_{1}\right)=k_{2}\left(y_{2}-y_{1}\right)+k_{2} \delta$

This leads to following system:

$$
\left\{\begin{array}{l}
k_{1} y_{1}-k_{2}\left(y_{2}-y_{1}\right)-k_{2} \delta+M g=0 \\
k_{2}\left(y_{2}-y_{1}\right)+k_{2} \delta+m g=0
\end{array}\right.
$$

so,

$$
\left\{\begin{array}{l}
y_{10}=-\frac{M g}{k_{1}}(1+\varepsilon)=-\gamma(1+\varepsilon) \\
y_{20}=-\frac{M g}{k_{1}}(1+\varepsilon)-\delta-\frac{m g}{k_{2}}=-\gamma(1+\varepsilon)-\delta-\varepsilon \frac{M g}{k_{2}}
\end{array}\right.
$$

This equilibrium point should be considered in all numerical results. We can impose equilibrium states to the numerical results as follows:

$$
\left\{\begin{array}{l}
v_{\text {numerical }}=\left(y_{1}-y_{10}\right)+\varepsilon\left(y_{2}-y_{20}\right) \\
w_{\text {numerical }}=\left(y_{1}-y_{10}\right)-\left(y_{2}-y_{20}\right)
\end{array}\right.
$$

So, $N_{1}$ and $N_{2}$ can be defined in terms of original system of equations $\left(N_{1}^{\text {exact }}\right.$ and $N_{2}^{\text {exact }}$ ) as:

$$
\begin{aligned}
& N_{1}^{\text {exact }}=\sqrt{\left(v_{\text {numerical }}\right)^{2}+\left(\dot{y_{1}}+\varepsilon \dot{y_{2}}\right)^{2}} . \\
& N_{2}^{\text {exact }}=\sqrt{\left(\text { w }_{\text {numerical }}\right)^{2}+\left(\dot{y_{1}}-\dot{y_{2}}\right)^{2}} .
\end{aligned}
$$


Let us consider the following initial conditions for the system:

$$
\begin{cases}y_{1}(0)=1.5+y_{10}, & \dot{y}_{1}(0)=0 \\ y_{2}(0)=y_{20}, & \dot{y}_{2}(0)=0\end{cases}
$$

Figure 4 presents $\tau_{0}$-invariant of the system and corresponding numerical results which are obtained by direct integration of (5) with the external forcing amplitude $f_{0}=0.1$. When the system arrives to the unstable zone, it tries to reach other stable zone by an abrupt jump between its stable branches through a bifurcation. This bifurcation leads the master structure to experience very low amplitude compared to initial stages of the vibration (see Fig. 5).

\subsection{Strongly Modulated Response in the Presence of Gravity}

The strongly modulated response (SMR) of the system in the vicinity of the 1:1 resonance is characterized by relaxation oscillations between stable branches of the slow invariant manifold, i.e. switches between slow motions at stable critical manifolds of the system and fast jumps between them. Starosvetsky and Gendelman [21] pinpointed this behavior in two coupled oscillators with essential cubic nonlinearity for the NES while Lamarque et al. [46] investigated the same behavior in systems with non-smooth NES. Let us consider (29) and (30). The possible relaxation of the system can occur if the flow in the vicinity of the lower fold line, i.e. $N_{21}$, experiences some bifurcation, i.e. for some points at the lower fold, $N_{2}^{\prime}$ changes its direction, so phase trajectories of the lower stable branch can change their direction and aim at

Fig. $4 \tau_{0}$-invariant manifold of the system in the presence of the gravity and corresponding numerical result with $f_{0}=0.1$. Numerical results are obtained by direct integration of (5)

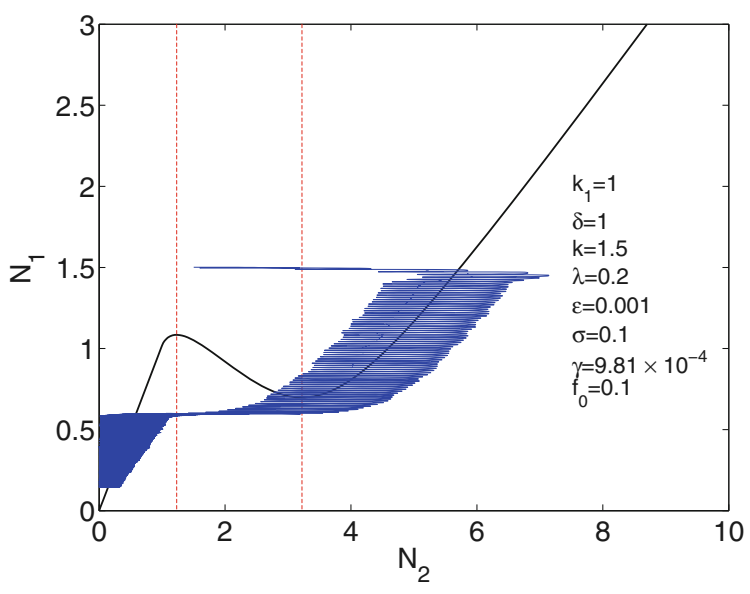


Fig. 5 Variation of system amplitudes with respect to the time $\left(f_{0}=0.1\right)$ : $\mathbf{a} N_{2} ; \mathbf{b}$ $N_{1}$. Results are obtained by direct integration of (5)

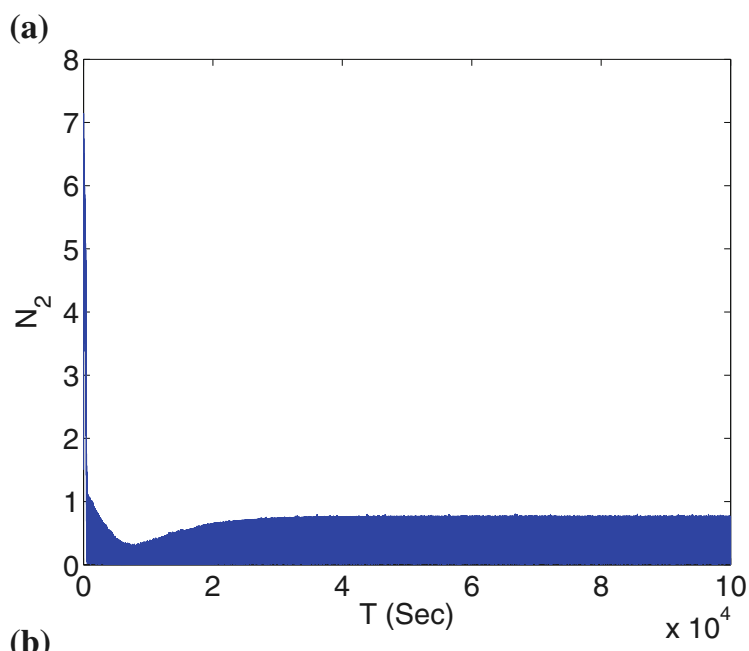

(b)

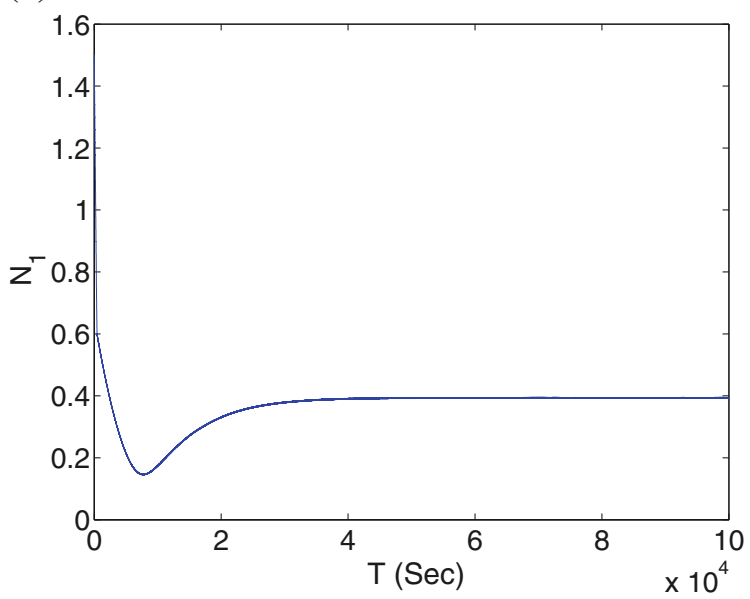

the fold line $N_{21}$ for a jump to upper stable branch. This criterion will be satisfied if in (29) and (30):

$$
\begin{aligned}
& f_{1}\left(N_{2}, \delta_{2}\right)=0 \\
& f_{2}\left(N_{2}, \delta_{2}\right)=0
\end{aligned}
$$

which corresponds to ordinary fixed points of the system under consideration. If in addition to this, $g\left(N_{2}\right)=0$ (the denominator of (29)) then the system will have fold singularities, i.e. singularity and equilibrium points coincide, so $N_{2}=N_{21}$ and $N_{2}=N_{22}$. It is worthwhile to mention that $g\left(N_{2}\right)=0$ is exactly equivalent to stability borders which are presented by (26) [48], i.e. 


$$
\lambda^{2}+\left(1-G_{f}\left(N_{2}^{2}\right)\right)\left(1-H\left(N_{2}^{2}\right) N_{2}-G_{f}\left(N_{2}^{2}\right)\right)=0
$$

Lamarque et al. [46] proved that necessary forcing condition for existence of the first pair of folded singularities, i.e. $\left(N_{21}, \delta_{21}\right)$, is:

$$
f_{0} \geq f_{0(1 \text { critical })}=\frac{\lambda N_{21}}{\sqrt{\lambda^{2}+\left(1-G_{f}\left(N_{21}^{2}\right)\right)^{2}}}
$$

and the second pair of folded singularities on the second fold, i.e. $\left(N_{22}, \delta_{21}\right)$ and $\left(N_{22}, \delta_{22}\right)$, exists if:

$$
f_{0} \geq f_{0(2 \text { critical })}=\frac{\lambda N_{22}}{\sqrt{\lambda^{2}+\left(1-G_{f}\left(N_{22}^{2}\right)\right)^{2}}}
$$

Let us analyze the system under external forcing term $f_{0}=0.3>f_{0}(1 \mathrm{critical})=$ 0.254. The global behavior of the system is depicted in Fig. 6 while histories of system amplitudes are illustrated in Fig. 7. The response of the system is strongly modulated by trapping into hysteresis loops and bifurcations between its stables branches. In order to clarify this, phase portraits of the system is depicted in Fig. 8a while a zoomed area of this figure is illustrated in Fig. 8b. It is seen that some flow lines change their direction toward the fold line $N_{21}$ which gives a hint of the relaxation of the system by facing to folded singularities in the form of saddle and node (see Fig. $8 \mathrm{~b}$ ).

Fig. $6 \tau_{0}$-invariant manifold of the system in the presence of the gravity and corresponding numerical result with $f_{0}=0.3$.

Numerical results are obtained by direct integration of (5)

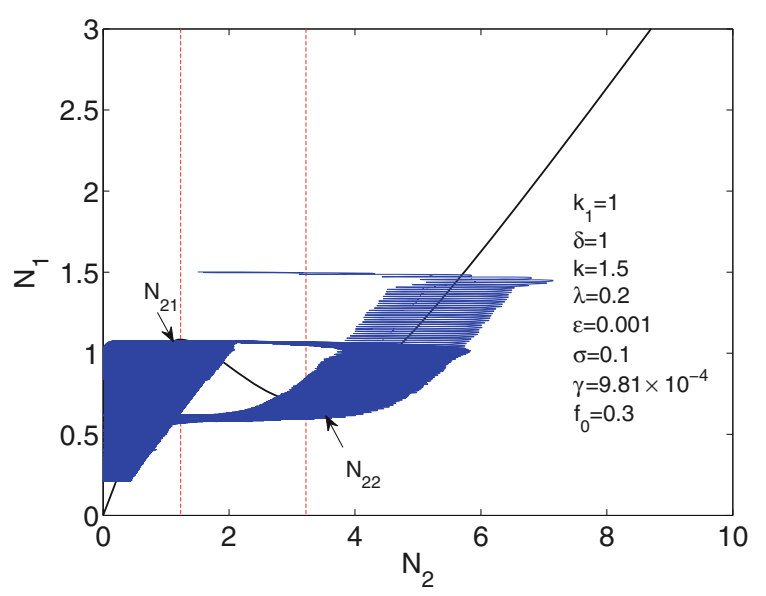


Fig. 7 SMR and Beating response of two oscillators with external forcing term $f_{0}=0.3>f_{0(1 \text { ritical })}=$ 0.254: a) $N_{2}$; b) $N_{1}$. Results are obtained by direct integration of (5)

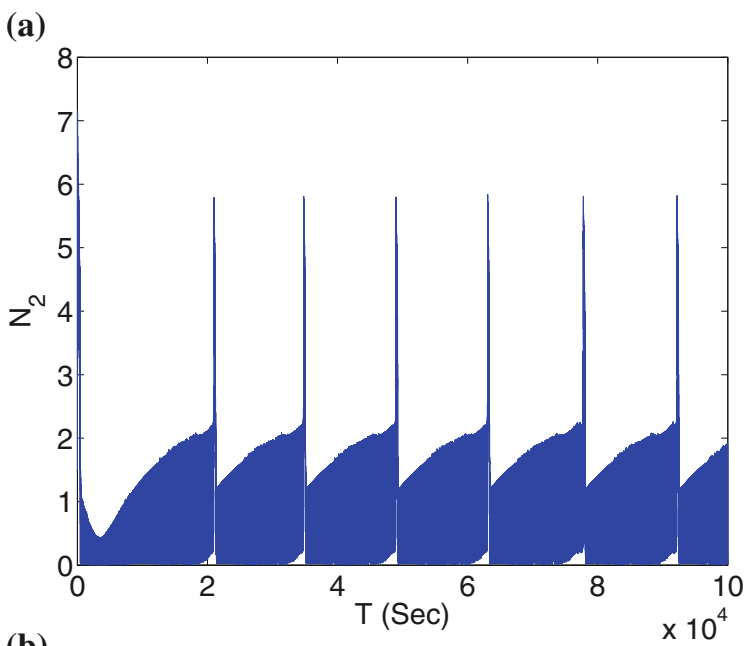

(b)

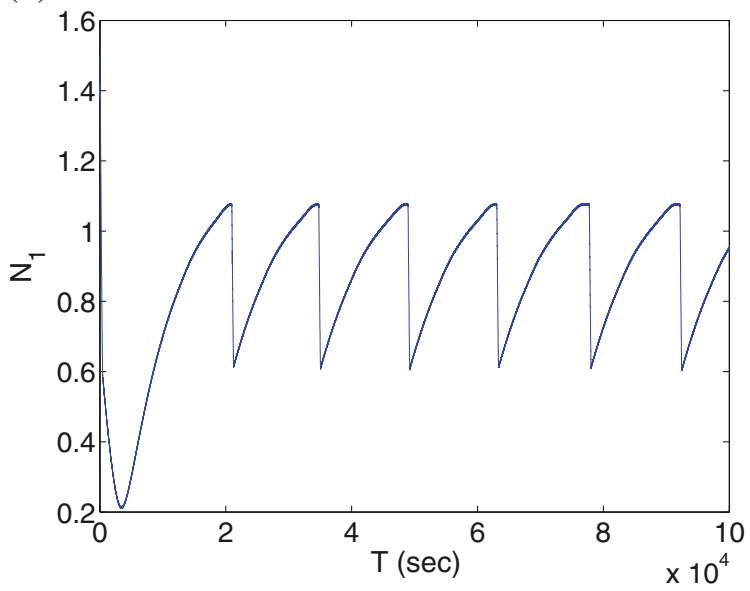

\section{Localization of Vibratory Energy of a Main System with a Set of Saint-Venant Elements by a NES with General Nonlinearity}

\subsection{Representation of the System}

We consider the system which is depicted in Fig. 9: It consists of two coupled oscillators. The first one with mass, stiffness and damping as $M, k_{0}$ and $\tilde{\lambda}$, which possesses a set of parallel Saint-Venant elements with characteristics as $\tilde{k}_{j}$ (stiffness) and $\alpha_{j}$ (threshold of the Saint-Venant element), $j=1,2, \ldots, n$. Each Saint-Venant element has an internal variable (displacement $u_{j}$ ). The second oscillator, namely NES has 
Fig. 8 Phase portrait for the system with $f_{0}=0.3>$ $f_{0(1 \text { critical })}=0.254$, a the overall view; $\mathbf{b}$ the zoomed area around folded singularities

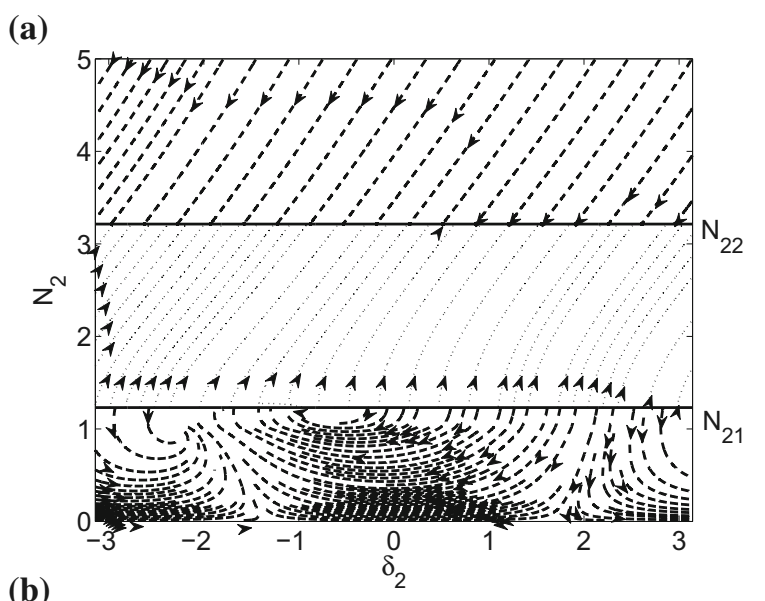

(b)

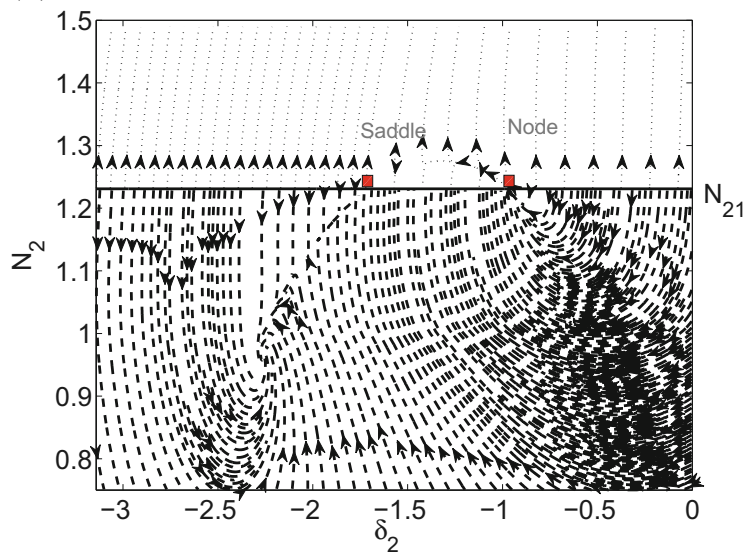

the mass, stiffness and damping as $m\left(0<\varepsilon=\frac{m}{M} \ll 1\right), \tilde{c}_{1}$ and $\tilde{\lambda}_{1}$. The potential of the NES $(F)$ is supposed to be a general "nonlinear" and "odd" function, i.e. $F(-z)=-F(z)$ (e.g. $F(z)=z^{3}$ ) [56]. If $x$ (versus $y$ ) be the displacement of the mass $M$ (respectively mass $m$ ), governing equations of the system can be summarized as:

$$
\left\{\begin{array}{l}
M \frac{d^{2} x}{d t^{2}}+\tilde{\lambda} \frac{d x}{d t}+\tilde{\lambda}_{1}\left(\frac{d x}{d t}-\frac{d y}{d t}\right)+k_{0} x+\sum_{j=1}^{n} \tilde{k}_{j} u_{j}+\tilde{c}_{1} F(x-y)=f_{1}(t) \\
m \frac{d^{2} y}{d t^{2}}+\tilde{\lambda}_{1}\left(\frac{d y}{d t}-\frac{d x}{d t}\right)+\tilde{c}_{1} F(y-x)=0 \\
\left(\frac{d u_{j}}{d t}+\beta\left(\frac{u_{j}}{\eta_{j}}\right)\right) \ni \frac{d x}{d t}, \quad \eta_{j}=\frac{\alpha_{j}}{\tilde{k}_{j}}, \quad j=1,2, \ldots, n
\end{array}\right.
$$




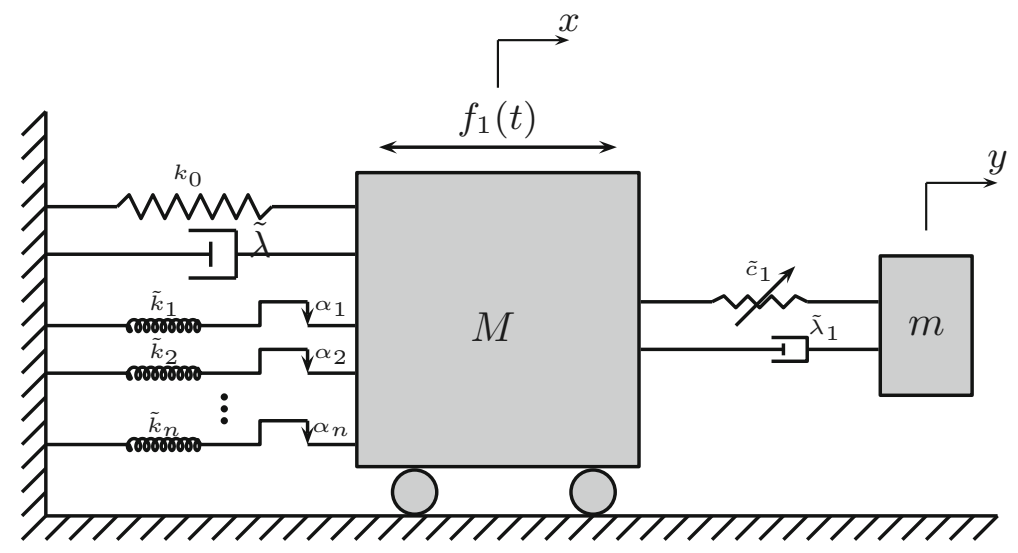

Fig. 9 Two coupled oscillators: the first one with a set of parallel Saint-Venant elements and under external force $f_{1}(t)$; the second one with general and odd nonlinear potential function $(m=\varepsilon M$, $0<\varepsilon \ll 1)$

Fig. 10 The $\beta$ graph in

Saint-Venant element

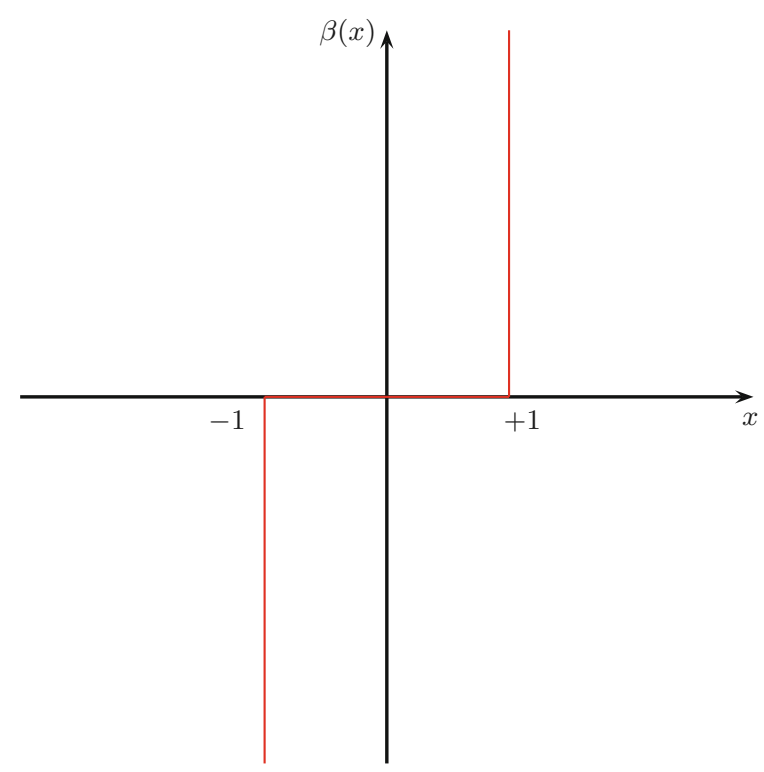

The $\beta$ graph which is depicted in Fig. 10 can be described as it follows:

$$
\beta(x)= \begin{cases}\varnothing & \text { if } x \in]-\infty,-1[\cup] 1,+\infty[ \\ 0 & \text { if } x \in]-1,1[ \\ \mathbb{R}_{-} \text {if } x=-1 & \mathbb{R}_{+} \text {if } x=1\end{cases}
$$


Let us introduce $\tau=t \sqrt{\frac{k_{0}}{M}}=\vartheta t, \frac{\tilde{\lambda} \vartheta}{M \vartheta^{2}}=\varepsilon \lambda_{0}, \frac{\tilde{k}_{j}}{M \vartheta^{2}}=\varepsilon k_{j}, \frac{\tilde{c}_{1}}{M \vartheta^{2}}=\varepsilon c_{10}$, $\frac{\tilde{\lambda}_{1} \vartheta}{M \vartheta^{2}}=\varepsilon \lambda_{10}, \frac{f_{1}\left(\frac{\tau}{\vartheta}\right)}{M \vartheta^{2}}=\varepsilon f_{10} \sin (\Omega \tau)$.

We mention that the differential inclusions of the model under consideration come from basic constitutive equations of the Saint-Venant elements as:

$$
k_{j} u_{j} \in \alpha_{j} \sigma\left(\frac{d x}{d t}-\frac{d u_{j}}{d t}\right), \quad j=1,2, \ldots, n
$$

where $\sigma$ is the graph of the sign:

$$
\sigma(z)= \begin{cases}-1 & \text { if } z<0 \\ {[-1,1]} & \text { if } z=0 \\ 1 & \text { if } z>0\end{cases}
$$

So, one should take into account that $\frac{d .}{d t}=\vartheta \frac{d}{d \tau}$ and

$$
\begin{aligned}
\tilde{k}_{j} u_{j} & \in \alpha_{j} \sigma\left(\vartheta\left(\frac{d x}{d \tau}-\frac{d u_{j}}{d \tau}\right)\right) \quad j=1,2, \ldots, n \\
\Leftrightarrow \tilde{k}_{j} u_{j} \in \alpha_{j} \sigma\left(\left(\frac{d x}{d \tau}-\frac{d u_{j}}{d \tau}\right)\right) \quad j & =1,2, \ldots, n
\end{aligned}
$$

Finally (47) are equivalent to

$$
\left\{\begin{array}{l}
\frac{d^{2} x}{d \tau^{2}}+\varepsilon \lambda_{0} \frac{d x}{d \tau}+\varepsilon \lambda_{10}\left(\frac{d x}{d \tau}-\frac{d y}{d \tau}\right)+x+\varepsilon \sum_{j=1}^{n} k_{j} u_{j}+\varepsilon c_{10} F(x-y) \\
=\varepsilon f_{10} \sin (\Omega \tau) \\
\varepsilon \frac{d^{2} y}{d \tau^{2}}+\varepsilon \lambda_{10}\left(\frac{d y}{d \tau}-\frac{d x}{d \tau}\right)+\varepsilon c_{10} F(y-x)=0 \\
\left(\frac{d u_{j}}{d \tau}+\beta\left(\frac{u_{j}}{\eta_{j}}\right)\right) \ni \frac{d x}{d \tau}, \quad \eta_{j}=\frac{\alpha_{j}}{\tilde{k}_{j}}, \quad j=1,2, \ldots, n
\end{array}\right.
$$


Let us introduce coordinates of the center of mass and relative displacement via

$$
\left\{\begin{array} { l } 
{ v = x + \varepsilon y } \\
{ w = x - y }
\end{array} \Leftrightarrow \left\{\begin{array}{l}
x=\frac{v+\varepsilon w}{1+\varepsilon} \\
y=\frac{v-w}{1+\varepsilon}
\end{array}\right.\right.
$$

System (52) becomes:

$$
\left\{\begin{array}{l}
\frac{d^{2} v}{d \tau^{2}}+\frac{\varepsilon \lambda_{0}}{1+\varepsilon}\left(\frac{d v}{d \tau}+\varepsilon \frac{d w}{d \tau}\right)+\frac{v+\varepsilon w}{1+\varepsilon}++\varepsilon \sum_{j=1}^{n} k_{j} u_{j}=\varepsilon f_{10} \sin (\Omega \tau) \\
\frac{d^{2} w}{d \tau^{2}}+\frac{\varepsilon \lambda_{0}}{1+\varepsilon}\left(\frac{d v}{d \tau}+\varepsilon \frac{d w}{d \tau}\right)+\frac{v+\varepsilon w}{1+\varepsilon}++\varepsilon \sum_{j=1}^{n} k_{j} u_{j} \\
+(1+\varepsilon)\left(\lambda_{10} \frac{d w}{d \tau}+c_{10} F(w)\right)=\varepsilon f_{10} \sin (\Omega \tau) \\
\left(\frac{d u_{j}}{d \tau}+\beta\left(\frac{u_{j}}{\eta_{j}}\right)\right) \ni \frac{1}{1+\varepsilon}\left(\frac{d v}{d \tau}+\varepsilon \frac{d w}{d \tau}\right), \quad \eta_{j}=\frac{\alpha_{j}}{\tilde{k}_{j}}, \quad j=1,2, \ldots, n
\end{array}\right.
$$

\subsection{Dynamical Behavior Around 1:1 Resonance}

Let us set $T=\Omega \tau$ and $\cdot=\frac{d}{d \tau}$. We introduce the following complex variables [57] to the system:

$$
\begin{array}{ll}
\phi_{1} e^{i T}=\Omega(\dot{v}+i v) & , \phi_{1}^{*} e^{-i T}=\Omega(\dot{v}-i v) \\
\phi_{2} e^{i T}=\Omega(\dot{w}+i w) & , \phi_{2}^{*} e^{-i T}=\Omega(\dot{w}-i w) \\
\phi_{2+j} e^{i T}=\Omega\left(\dot{u}_{j}+i u_{j}\right), & \phi_{2+j}^{*} e^{-i T}=\Omega\left(\dot{u}_{j}-i u_{j}\right), j=1,2, \ldots, n
\end{array}
$$

with $i^{2}=-1$. To investigate the $1: 1$ resonance, we assume $\Omega=1+\sigma \varepsilon$.

We consider only equations obtained by Galerkin method and truncated Fourier series: Indeed we take into account only first harmonic $e^{i T}$ for each equation. To calculate the corresponding Fourier coefficients we assume that $\phi_{l}$ and $\phi_{l}^{*}(l=$ $1,2, \ldots, n+j)$ do not depend on $T$. We will either verify this assumption during the multiple scales analysis, or we will assume that after a transient long enough $\phi_{l}$ and $\phi_{l}^{*}(l=1,2, \ldots, n+j)$ reach to an "asymptotic state" independently of $T$. Nevertheless we also keep $\dot{\phi}_{l}$ and $\dot{\phi}_{2}$ in the equations. Then we obtain following system: 


$$
\begin{aligned}
& \Omega \dot{\phi}_{1}-\frac{\Omega}{2 i} \phi_{1}+\frac{\varepsilon \lambda_{0}\left(\phi_{1}+\varepsilon \phi_{2}\right)}{2(1+\varepsilon)}+\frac{\phi_{1}+\varepsilon \phi_{2}}{2 i \Omega(1+\varepsilon)}+\varepsilon \frac{\sum_{j=1}^{n} k_{j} \phi_{j+2}}{2 \Omega i}=\varepsilon \frac{f_{10}}{2 i} \\
& \Omega \dot{\phi}_{2}-\frac{\Omega}{2 i} \phi_{2}+\frac{\varepsilon \lambda_{0}\left(\phi_{1}+\varepsilon \phi_{2}\right)}{2(1+\varepsilon)}+\frac{\phi_{1}+\varepsilon \phi_{2}}{2 i \Omega(1+\varepsilon)}+\frac{\sum_{j=1}^{n} k_{j} \phi_{j+2}}{2 \Omega i} \\
& +(1+\varepsilon)\left(c_{10} \mathscr{F}+\frac{\lambda_{10}}{2} \phi_{2}\right)=\varepsilon \frac{f_{10}}{2 i} \\
& \phi_{j+2}=\frac{\phi_{1}+\varepsilon \phi_{2}}{(1+\varepsilon) \pi} \xi_{j}\left(\frac{\left|\phi_{1}+\varepsilon \phi_{2}\right|}{(1+\varepsilon) \Omega}\right), \quad j=1,2, \ldots, n
\end{aligned}
$$

where

$$
\mathscr{F}=\frac{1}{2 \pi} \int_{0}^{2 \pi} e^{-i T} F\left(\frac{\phi_{1} e^{i T}-\phi_{2}^{*} e^{-i T}}{2 i \Omega}\right) \mathrm{d} T
$$

and $\xi_{j}(z)\left(\forall z \in \mathbb{R}_{+}, j=1,2, \ldots, n\right)$ reads:

$$
\xi_{j}(z)= \begin{cases}\pi & \text { if } z \leqslant \eta_{j} \\ \theta+e^{-i \theta} \sin (\theta)-4 e^{-i \frac{\theta}{2}} \sin \left(\frac{\theta}{2}\right)-\frac{4 \eta_{j}}{z} e^{-i\left(\theta+\frac{\pi}{2}\right)} & \text { if } z>\eta_{j}\end{cases}
$$

with

$$
\theta=\arccos \left(1-\frac{2 \eta_{j}}{z}\right)
$$

As in the Sect. 3 a multiple scale approach [58] with a small (and given) parameter $\varepsilon$ is presented by considering fast time $T_{0}=T$, and slow times $T_{l}=\varepsilon^{l} T, l=1,2, \ldots$ so that:

$$
\frac{d}{d T}=\frac{d}{d T_{0}}+\varepsilon \frac{d}{d T_{1}}+\varepsilon^{2} \frac{d}{d T_{2}}+\ldots
$$

\subsection{1 $\varepsilon^{0}$-Order of the System}

At $\varepsilon^{0}$ order, following equations can be derived from the system of (56):

$$
\begin{gathered}
\frac{\partial \phi_{1}}{\partial T_{0}}=0 \Rightarrow \phi_{1}=\phi_{1}\left(T_{1}, T_{2}, \ldots\right) \\
\frac{\partial \phi_{2}}{\partial T_{0}}+\frac{\phi_{1}-\phi_{2}}{2 i}+c_{10} \mathscr{F}+\frac{\lambda_{10}}{2} \phi_{2}=0
\end{gathered}
$$




$$
\phi_{j+2}=\frac{\phi_{1}}{\pi} \xi_{j}\left(\left|\phi_{1}\right|\right), \quad j=1,2, \ldots, n
$$

We can see from equations that $\phi_{1}$ is a constant versus $T_{0}=T$, as well as $\phi_{j+2}$, $j=1,2, \ldots, n$, so the assumption for calculation of Fourier coefficients of $e^{i T}=$ $e^{i T_{0}}$ is verified a posteriori. For $\phi_{2}$, we can not claim the same property. This is why we process as follows: We assume that when $T_{0} \rightarrow \infty, \phi_{2}$ reaches an asymptotic equilibrium governed by a manifold called $T_{0}$-invariant. Then we have:

$$
\frac{\phi_{1}-\phi_{2}}{2 i}+c_{10} \mathscr{F}+\frac{\lambda_{10}}{2} \phi_{2}=0
$$

so that implicitly $\phi_{2}$ may depend on $T_{1}$ now, but no longer on $T_{0}$ (after $T_{0}$ long enough to approach the asymptotic state of $\phi_{2}$ governed by (64)). We study modulation of the dynamics around periodic solution depending on time $T_{0}$ associated to the $T_{0}$ invariant. Let us also notice that equations for $\phi_{j+2}, j=1,2, \ldots, n$ are governed by first order differential equations.

\subsection{2 $\varepsilon^{1}$-Order of the System and Modulations Around $T_{0}$-Invariant}

The $\varepsilon^{1}$ order of the first equation of system (56) reads:

$$
\frac{d \phi_{1}}{d T_{1}}+\frac{\lambda_{0}}{2} \phi_{1}+\frac{\phi_{2}}{2 i}-\frac{2 \sigma+1}{2 i} \phi_{1}+\frac{\sum_{j=1}^{n} k_{j} \phi_{j+2}}{2 i}=\frac{f_{10}}{2 i}
$$

Let us consider $T_{0}$-invariant and also obtained solutions at $\varepsilon^{0}$ order for $\phi_{j+2}, j=$ $1,2, \ldots, n$. We write (64) in the general form:

$$
\phi_{1}=H\left(\phi_{2}, \phi_{2}^{*}\right)
$$

We introduce polar form for $\phi_{j}, j=1,2, \ldots, n+2$ as it follows:

$$
\phi_{j}=N_{j} e^{i \delta_{j}}, N_{j} \in \mathbb{R}_{+}, \delta_{j} \in \mathbb{R}
$$

From relation (66) it is clear that we can obtain two explicit analytical solutions providing $N_{1}$ and $\delta_{1}$ as functions of $N_{2}$ and $\delta_{2}$ :

$$
\begin{aligned}
& N_{1}=H_{1}\left(N_{2}, \delta_{2}\right) \\
& \delta_{1}=H_{2}\left(N_{2}, \delta_{2}\right)
\end{aligned}
$$


From the (63) we have:

$$
N_{j+2} e^{i \delta_{j+2}}=\frac{N_{1}}{\pi} e^{i \delta_{1}} \xi_{j}\left(N_{1}\right), \quad j=1,2, \ldots, n
$$

or

$$
N_{j+2} e^{i\left(\delta_{j+2}-\delta_{1}\right)}=\frac{N_{1}}{\pi} \xi_{j}\left(N_{1}\right), \quad j=1,2, \ldots, n
$$

so that

$$
N_{j+2}=\frac{N_{1}}{\pi}\left|\xi_{j}\left(N_{1}\right)\right|, \quad j=1,2, \ldots, n
$$

and $\delta_{j+2}$ depends on $N_{1}$ and $\delta_{1}$. Let us write

$$
\delta_{j+2}=\rho_{j}\left(N_{1}, \delta_{1}\right), \quad j=1,2, \ldots, n
$$

From (65) we have:

$$
\frac{\partial N_{1}}{\partial T_{1}}+i N_{1} \frac{\partial \delta_{1}}{\partial T_{1}}+\left(\frac{\lambda_{0}}{2}-\frac{2 \sigma+1}{2 i}\right) N_{1}+\frac{N_{2}}{2 i} e^{i\left(\delta_{2}-\delta_{1}\right)}+\frac{\sum_{j=1}^{n} k_{j} \frac{N_{1}}{\pi} \xi_{j}\left(N_{1}\right)}{2 i}=\frac{f_{10}}{2 i} e^{-i \delta_{1}}
$$

Introducing real and imaginary parts of $\xi$

$$
\xi_{j}\left(N_{1}\right)=\xi_{j r}\left(N_{1}\right)+i \xi_{j i}\left(N_{1}\right), \quad j=1,2, \ldots, n
$$

finally one can obtain:

$$
\left\{\begin{array}{l}
\frac{\partial N_{1}}{\partial T_{1}}+\frac{\lambda_{0}}{2} N_{1}+\frac{N_{2}}{2} \sin \left(\delta_{2}-\delta_{1}\right)+\frac{\sum_{j=1}^{n} k_{j} \frac{N_{1}}{\pi} \xi_{j i}\left(N_{1}\right)}{2}=-\frac{f_{10}}{2} \sin \left(\delta_{1}\right) \\
N_{1} \frac{\partial \delta_{1}}{\partial T_{1}}+\frac{2 \sigma+1}{2} N_{1}-\frac{N_{2}}{2} \cos \left(\delta_{2}-\delta_{1}\right)-\frac{\sum_{j=1}^{n} k_{j} \frac{N_{1}}{\pi} \xi_{j r}\left(N_{1}\right)}{2}=-\frac{f_{10}}{2} \cos \left(\delta_{1}\right)
\end{array}\right.
$$

Then, from (68) we can reach a linear system in $\frac{\partial N_{2}}{\partial T_{1}}$ and $\frac{\partial \delta_{2}}{\partial T_{1}}$ :

$$
\left\{\begin{array}{l}
\frac{\partial H_{1}}{\partial N_{2}} \frac{\partial N_{2}}{\partial T_{1}}+\frac{\partial H_{1}}{\partial \delta_{2}} \frac{\partial \delta_{2}}{\partial T_{1}}-m_{1}=0 \\
H_{1}\left(\frac{\partial H_{2}}{\partial N_{2}} \frac{\partial N_{2}}{\partial T_{1}}+\frac{\partial H_{2}}{\partial \delta_{2}} \frac{\partial \delta_{2}}{\partial T_{1}}\right)-m_{2}=0
\end{array}\right.
$$


where

$$
\begin{aligned}
& -m_{1}=\frac{\lambda_{0}}{2} H_{1}+\frac{N_{2}}{2} \sin \left(\delta_{2}-H_{2}\right)+\frac{\sum_{j=1}^{n} k_{j} \frac{H_{1}}{\pi} \xi_{j i}\left(H_{1}\right)}{2}+\frac{f_{10}}{2} \sin \left(\delta_{1}\right) \\
& -m_{2}=\frac{2 \sigma+1}{2} H_{1}-\frac{N_{2}}{2} \cos \left(\delta_{2}-H_{2}\right)-\frac{\sum_{j=1}^{n} k_{j} \frac{H_{1}}{\pi} \xi_{j r}\left(H_{1}\right)}{2}+\frac{f_{10}}{2} \cos \left(\delta_{1}\right)
\end{aligned}
$$

Finally, by solving the system (76), the following equations are obtained:

$$
\begin{array}{r}
\frac{\partial N_{2}}{\partial T_{1}}=\frac{\tilde{f}_{1}\left(N_{2}, \delta_{2}\right)}{\tilde{g}\left(N_{2}, \delta_{2}\right)} \\
N_{2} \frac{\partial \delta_{2}}{\partial T_{1}}=\frac{\tilde{f}_{2}\left(N_{2}, \delta_{2}\right)}{\tilde{g}\left(N_{2}, \delta_{2}\right)}
\end{array}
$$

where

$$
\begin{aligned}
& \tilde{f}_{1}\left(N_{2}, \delta_{2}\right)=H_{1} \frac{\partial H_{2}}{\partial \delta_{2}} m_{1}-\frac{\partial H_{1}}{\partial \delta_{2}} m_{2} \\
& \tilde{f}_{2}\left(N_{2}, \delta_{2}\right)=N_{2}\left(\frac{\partial H_{1}}{\partial N_{2}} m_{2}-H_{1} \frac{\partial H_{2}}{\partial N_{2}} m_{1}\right) \\
& \tilde{g}\left(N_{2}, \delta_{2}\right)=H_{1}\left(\frac{\partial H_{1}}{\partial N_{2}} \frac{\partial H_{2}}{\partial \delta_{2}}-\frac{\partial H_{2}}{\partial N_{2}} \frac{\partial H_{1}}{\partial \delta_{2}}\right)
\end{aligned}
$$

\subsection{Analysis of the Dynamics: General Method}

The analysis of the dynamical behavior corresponding to a modulation at 1:1 resonance around the $T_{0}$-invariant is given by:

- geometry of the $T_{0}$-invariant in the $N_{1}, N_{2}$ and $\delta_{2}$ space associated to the relation $N_{1}=H_{1}\left(N_{2}, \delta_{2}\right)$.

- equilibrium points of the reduced system (78) are given by:

$$
\left\{\begin{array}{l}
f_{1}\left(N_{2}, \delta_{2}\right)=0, f_{2}\left(N_{2}, \delta_{2}\right)=0 \\
g_{1}\left(N_{2}, \delta_{2}\right) \neq 0, g_{2}\left(N_{2}, \delta_{2}\right) \neq 0
\end{array}\right.
$$

if $f_{1}, f_{2}, g_{1}$ and $g_{2}$ correspond to numerators and denominators of the system (78). 
- singular points of the reduced system (78) are given by:

$$
\left\{\begin{array}{l}
f_{1}\left(N_{2}, \delta_{2}\right)=0, f_{2}\left(N_{2}, \delta_{2}\right)=0 \\
g_{1}\left(N_{2}, \delta_{2}\right)=0, g_{2}\left(N_{2}, \delta_{2}\right)=0
\end{array}\right.
$$

if $f_{1}, f_{2}, g_{1}$ and $g_{2}$ correspond to numerators and denominators of the system (78). Singular points are potentially associated to bifurcations.

\subsection{Analysis of the Dynamics for a Particular Case}

Let us choose $n=2$ and

$$
F(z)=z^{3}
$$

in such a case, we have

$$
\mathscr{F}=\frac{1}{2 i} G\left(\left|\phi_{2}\right|^{2}\right) \phi_{2}
$$

with

$$
G(\chi)=\frac{3}{4} \chi, \quad \chi \geq 0
$$

Then

$$
\begin{aligned}
\phi_{1}=H\left(\phi_{2}, \phi_{2}^{*}\right) & =\phi_{2}-2 i c_{10} \mathscr{F}-i \lambda_{10} \phi_{2} \\
& =\phi_{2}-\frac{3}{4} c_{10}\left|\phi_{2}\right|^{2} \phi_{2}-i \lambda_{10} \phi_{2}
\end{aligned}
$$

and

$$
\begin{aligned}
& H_{1}\left(N_{2}, \delta_{2}\right)=N_{2} \sqrt{\lambda_{10}^{2}+\left(1-\frac{3}{4} c_{10} N_{2}^{2}\right)^{2}} \\
& H_{2}\left(N_{2}, \delta_{2}\right)=\delta_{2}+\arctan \left(\frac{-\lambda_{10}}{1-\frac{3}{4} c_{10} N_{2}^{2}}\right)
\end{aligned}
$$

Now, $m_{1}$ and $m_{2}$ can be obtained from the general expression in (77). Moreover, we have simplified expression for $\tilde{g}$ since $H_{1}$ does not depend on $\delta_{2}$ and $H_{2}$ depends linearly on $\delta_{2}$.

$$
\tilde{g}\left(N_{2}, \delta_{2}\right)=H_{1} \frac{\partial H_{1}}{\partial N_{2}}
$$


From the expression of $H_{1}$ it is clear that $\tilde{g}$ does not depend on $\delta_{2}$. We have also:

$$
\begin{aligned}
& \tilde{f}_{1}\left(N_{2}, \delta_{2}\right)=H_{1} m_{1} \\
& \tilde{f}_{2}\left(N_{2}, \delta_{2}\right)=\left(\frac{\partial H_{1}}{\partial N_{2}} m_{2}-H_{1} \frac{\partial H_{2}}{\partial N_{2}} m_{1}\right) N_{2}
\end{aligned}
$$

Finally, let us give expressions of $f_{1}, f_{2}, g_{1}$ and $g_{2}$. The reduced system of equations reads:

$$
\begin{aligned}
\frac{\tilde{f}_{1}}{\tilde{g}} & =\frac{m_{1}}{\frac{\partial H_{1}}{\partial N_{2}}} \\
& =\frac{m_{1} \sqrt{\lambda_{10}^{2}+\left(1-\frac{3}{4} c_{10} N_{2}^{2}\right)^{2}}}{\lambda_{10}^{2}+\left(1-\frac{3}{4} c_{10} N_{2}^{2}\right)\left(1-\frac{9}{4} c_{10} N_{2}^{2}\right)} \\
\frac{\tilde{f_{2}}}{\tilde{g}} & =\frac{N_{2}\left(\frac{\partial H_{1}}{\partial N_{2}} m_{2}-H_{1} \frac{\partial H_{2}}{\partial N_{2}} m_{1}\right)}{H_{1} \frac{\partial H_{1}}{\partial N_{2}}} \\
& =\frac{3 \lambda_{10} c_{10} N_{2}^{2} m_{1}+2 m_{2}\left(\lambda_{10}^{2}+\left(1-\frac{3}{4} c_{10} N_{2}^{2}\right)\left(1-\frac{9}{4} c_{10} N_{2}^{2}\right)\right)}{2\left(\lambda_{10}^{2}+\left(1-\frac{3}{4} c_{10} N_{2}^{2}\right)\left(1-\frac{9}{4} c_{10} N_{2}^{2}\right)\right) \sqrt{\lambda_{10}^{2}+\left(1-\frac{3}{4} c_{10} N_{2}^{2}\right)\left(1-\frac{9}{4} c_{10} N_{2}^{2}\right)}}
\end{aligned}
$$

so that:

$$
\begin{aligned}
& f_{1}=m_{1} \sqrt{\lambda_{10}^{2}+\left(1-\frac{3}{4} c_{10} N_{2}^{2}\right)^{2}} \\
& f_{2}=3 \lambda_{10} c_{10} N_{2}^{2} m_{1}+2 m_{2}\left(\lambda_{10}^{2}+\left(1-\frac{3}{4} c_{10} N_{2}^{2}\right)\left(1-\frac{9}{4} c_{10} N_{2}^{2}\right)\right) \\
& g_{1}=\lambda_{10}^{2}+\left(1-\frac{3}{4} c_{10} N_{2}^{2}\right)\left(1-\frac{9}{4} c_{10} N_{2}^{2}\right) \\
& g_{2}=2 g_{1} \sqrt{\lambda_{10}^{2}+\left(1-\frac{3}{4} c_{10} N_{2}^{2}\right)^{2}}
\end{aligned}
$$

Equilibrium points are given by:

$$
f_{1}=0, f_{2}=0 \text { and } g_{1} \neq 0
$$

and singular points are governed by:

$$
f_{1}=0, f_{2}=0 \text { and } g_{1}=0
$$

This is equivalent to:

$$
\left\{\begin{array}{l}
m_{1}=0 \\
g_{1}=0
\end{array}\right.
$$


Then $g_{1}=0$ provides analytical values of $N_{2}$ (fold lines):

$$
N_{2}=\frac{2}{3} \sqrt{\frac{1}{c_{10}}\left(2 \mp \sqrt{1-3 \lambda_{10}^{2}}\right)}
$$

For a given $N_{2}, m_{1}$ depends only on the variable $\delta_{2}$ and can be solved numerically.

\subsection{Analytical Developments Versus Numerical Integrations}

Let us set $c_{10}=1, \lambda_{10}=0.1, \lambda_{0}=0.1, \eta_{1}=0.1, \eta_{2}=0.15, k_{1}=1, k_{2}=2$, $\varepsilon=0.001$. We consider that $f_{10}=0.7$. Euler's scheme $[54,59]$ with time steps as $\Delta \tau=10^{-4}$ is endowed for solving system of (52). Assumed initial conditions are $x(0)=0.5$ and $y(0)=\dot{x}(0)=\dot{y}(0)=u_{1}(0)=u_{2}(0)=0$.

Fig. 11 Positions of equilibrium points and fold singularities of the system with external forcing term $f_{10}=0.7$ (see (78) and (90)): $f_{1}=0(-), f_{2}=0$ $(---), g_{1}=0(-\cdot-\cdot-$, i.e. fold lines $N_{21}$ and $N_{22}$ ). The system possesses two fold singularities (no. 1 and 2) and three equilibrium points (no. 3, 4 and 5)

Fig. $12 T_{0}$-invariant of the system (solid blue line) and corresponding numerical results (black line) that are obtained by integration of (52) with external forcing term $f_{10}=0.7$
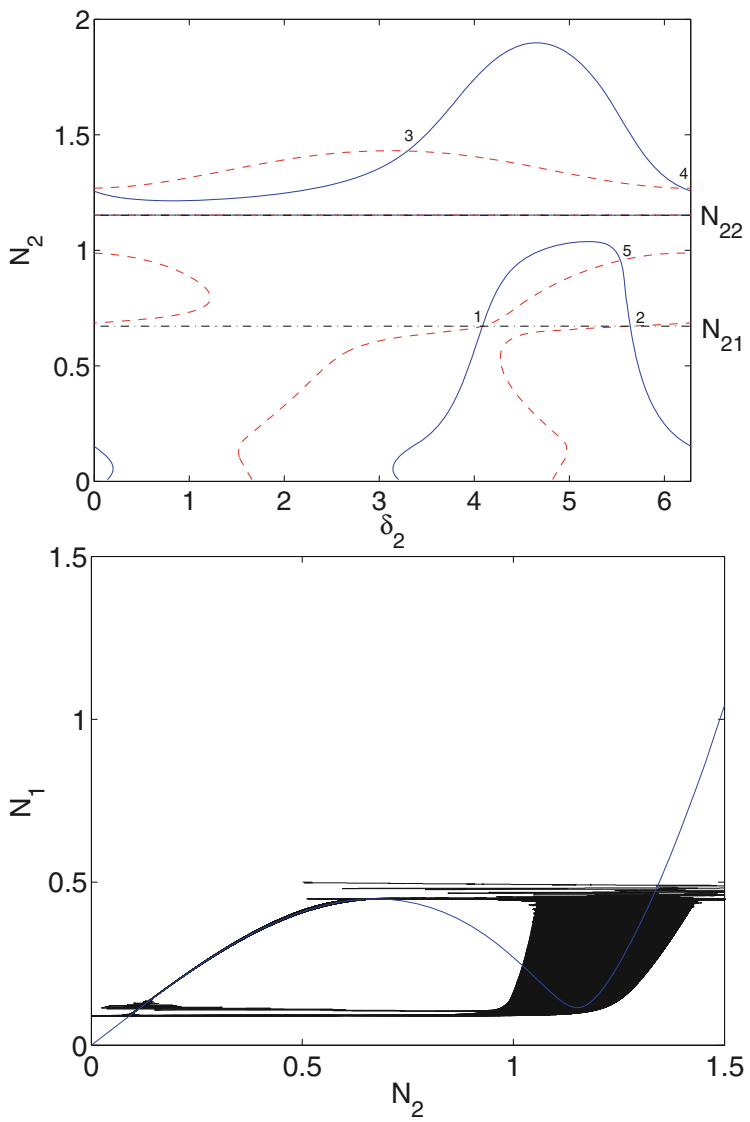
Fig. 13 Histories of system amplitudes that are obtained by integration of (52) with external forcing term $f_{10}=0.7: \mathbf{a} N_{1} ; \mathbf{b} N_{2}$ (a)

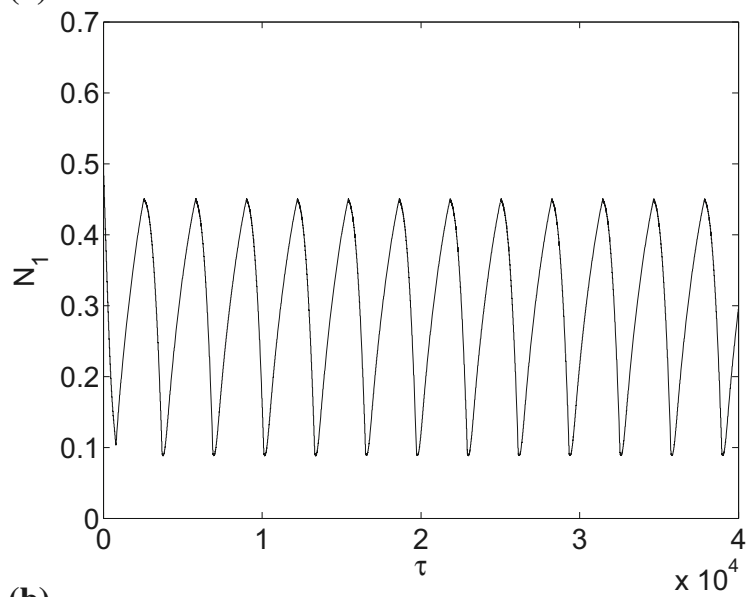

(b)

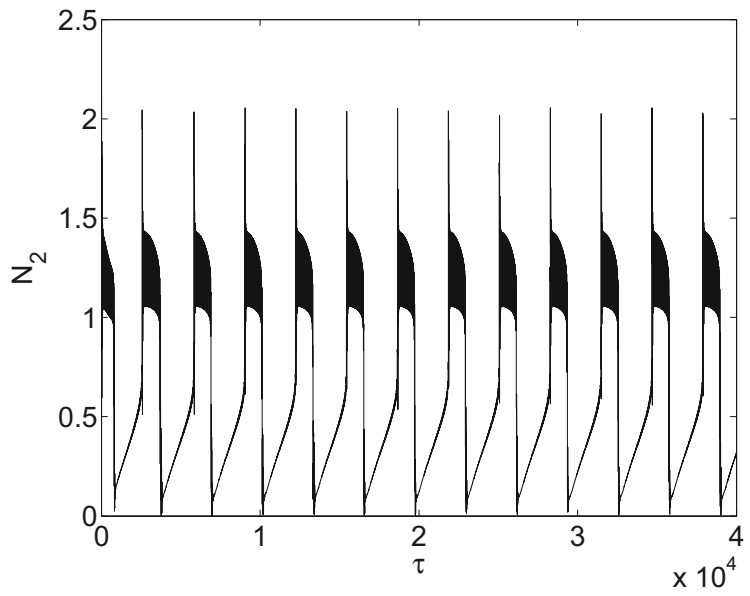

Predictions of all possible dynamics of the system until reaching the infinity of the $T_{1}$ time scale are shown in Fig. 11. It is seen that the system has two fold singularities on the first fold line $N_{21}$, namely points 1 and 2, two equilibrium points (no. 3 and no. 4) and another equilibrium point between two fold lines of the system (unstable area) namely point no. 5. $T_{0}$-Invariant of the system and corresponding numerical results are presented in Fig. 12. The system presents SMR by persisting direct and reverse bifurcations between its stability borders. This is due to the existence of fold singularities on fold line(s) of the system [21, 46]. This behavior will be more visible 
Fig. 14 Phase portraits of the reduced system with external forcing term $f_{10}=0.7$ (see (78), (89) and (90)) around the singular point no. 1 (saddle)

Fig. 15 Phase portraits of the reduced system with external forcing term $f_{10}=0.7$ (see (78), (89) and (90)) around the singular point no. 2 (node)
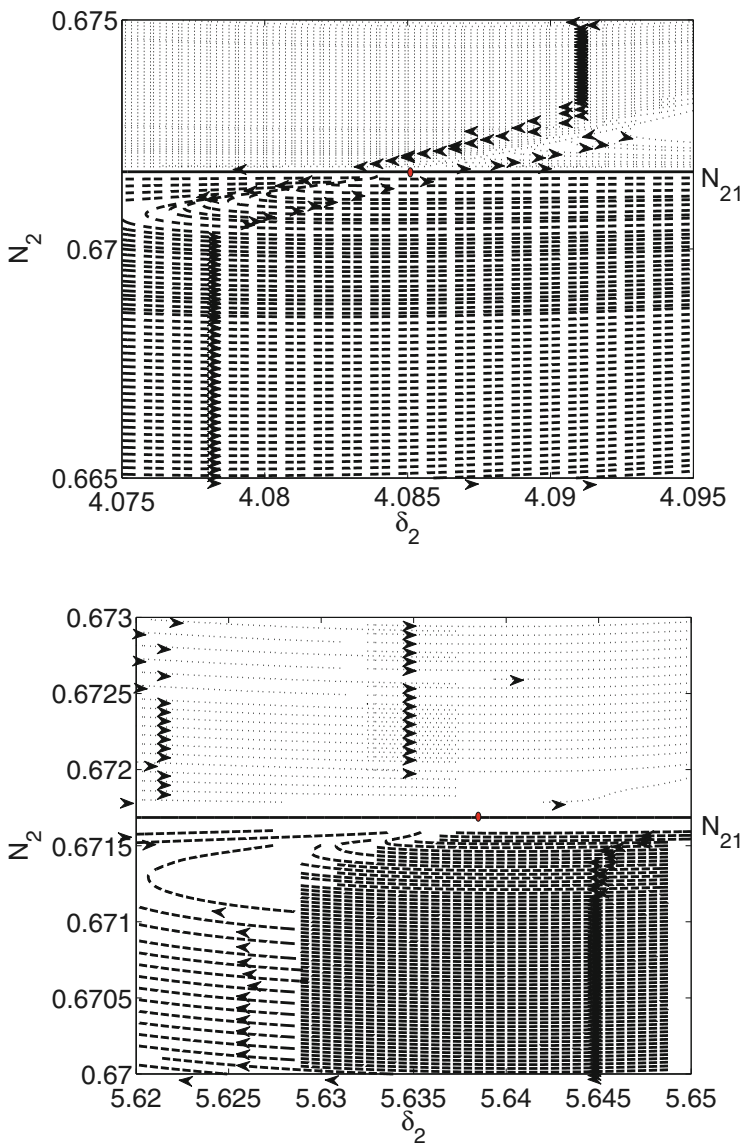

by looking at the histories of system amplitudes which are obtained by numerical integration and are illustrated in Fig. 13. Phase portraits of the reduced system (78) around singular points no. 1 and 2 are presented in Figs. 14 and 15 show that these singular points are in the form of saddle and nodes on the fold line of the system $\left(N_{21}\right)$. During SMR both oscillators and all of their components present beating responses: displacement histories of two oscillators which are depicted in Fig. 16 and also histories of internal variables of Saint-Venant elements that are presented in Fig. 17 show not only beating responses of all components of two oscillators during SMR but also activations of Saint-Venant elements during energy exchanges. The SMR of an optimized designed system is a very desirable behavior from passive control and also energy harvesting view points since both oscillators continue to exchange the energy with large intervals of energy changes for the NES and small 
Fig. 16 Displacements histories that are obtained by integration of (52) with external forcing term $f_{10}=0.7: \mathbf{a} x ; \mathbf{b} y$ (a)
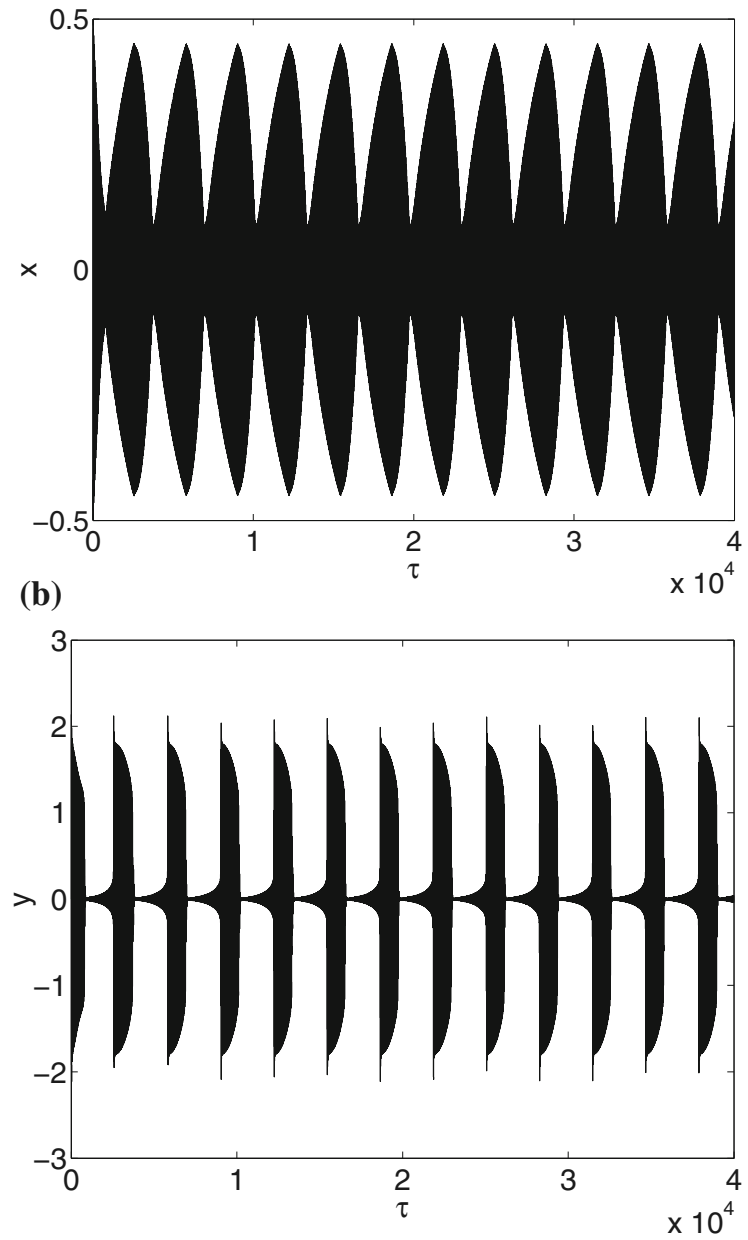

energy intervals for the main system. The system possesses two equilibrium points namely points no. 3 and 4 (see Fig. 11). It can be attracted by one of these points after a very long time at $T_{1}$ time scale or during higher time scales $\left(T_{2}, T_{3}, \ldots\right)$. Due to costly simulation time we did not run it for very long time scales. 
Fig. 17 Histories of internal variables of the Saint-Venant elements that are obtained by integration of (52) with external forcing term $f_{10}=0.7: \mathbf{a} u_{1} ; \mathbf{b} u_{2}$

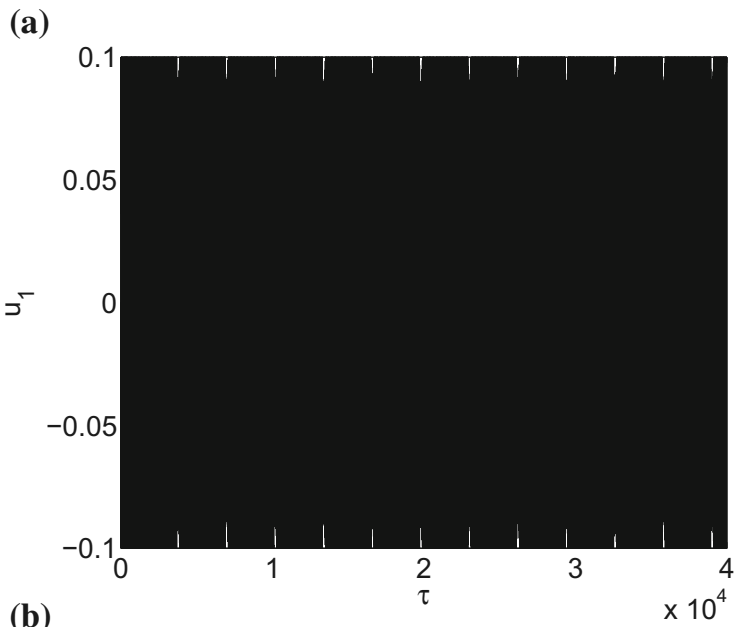

(b)

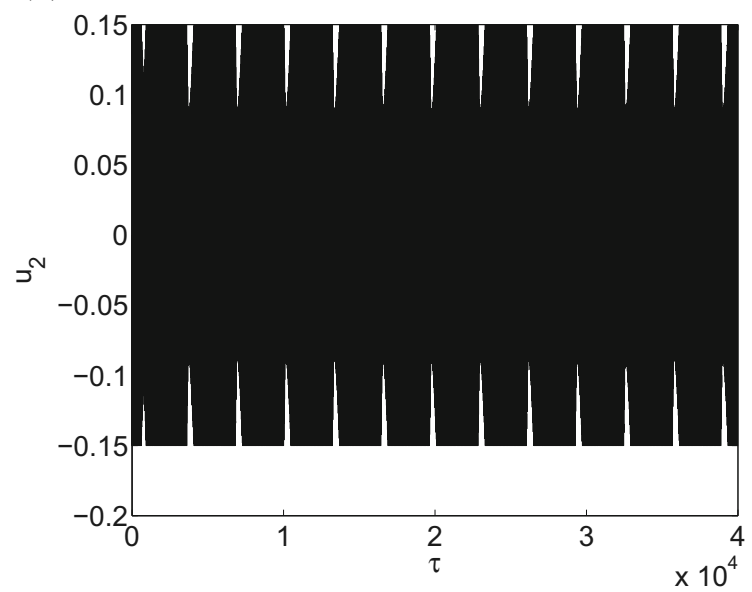

\section{Conclusions}

Multiple scale energy exchanges of two different coupled systems are considered: (I) a vertical system (i.e. consideration of effects of the gravity) which consists of a linear main structural system and a coupled nonsmooth nonlinear energy sink; (II) a main oscillator with a set of Saint-Venant elements that is coupled to a nonlinear energy sink with a general odd nonlinear potential function. Invariants of both systems and their geometries at fast time scale let us understand the process of energy exchanges between two oscillators with explanation of possible bifurcations between two coupled oscillators. Reduced form of equations of systems at slow time scale give us some tools to predict all possible regimes of systems during energy exchanges: systems can face periodic regimes due to existence of equilibrium points while they 
can present strongly modulated responses when fold singularities are present. These studies provide analytical design tools for tuning parameters of nonlinear energy sink according to the design goal which can be passive control of linear/nonlinear main structural systems by means of nonlinear energy sink. Experimentally realizations of both systems can be carried out by considering a moving light mass in a (frictionless) guide which is encased between two elastic walls at each end for representing a nonsmooth NES of the system I. Identification of parameters of Magnetorheological dampers which present hysteresis behaviors can lead to models represented by system II (with potentially added smooth nonlinear terms to the main structure) $[54,60]$.

Acknowledgments The authors would like to thank the following organizations for supporting this research work: (i) LABEX CELYA (ANR-10-LABX-0060) of the "Université de Lyon" within the program "Investissement d'Avenir" (ANR-11-IDEX-0007) operated by the French National Research Agency (ANR); (ii) Peugeot Citroën Automobiles OpenLab, Vibro-AcousticTribology@Lyon(VAT@Lyon).

\section{References}

1. Gendelman, O.V., Vakakis, A.F.: Transitions from localization to nonlocalization in strongly nonlinear damped oscillators. Chaos Soliton Frac. 11, 1535-1542 (2000)

2. Gendelman, O.V., Manevitch, L.I.: Reflection of short rectangular pulses in the ideal string attached to strongly nonlinear oscillator. Chaos Soliton Frac. 11, 2473-2477 (2000)

3. Gendelman, O.V.: Transition of energy to a nonlinear localized mode in a highly asymmetric system of two oscillators. Nonlinear Dyn. 25, 237-253 (2001)

4. Gendelman, O.V., Manevitch, L.I., Vakakis, A.F., M'Closkey, R.: Energy pumping in nonlinear mechanical oscillators: part I-dynamics of the underlying Hamiltonian systems. J. Appl. Mech. 68(1), 34-41 (2001)

5. Vakakis, A.F., Gendelman, O.V.: Energy pumping in nonlinear mechanical oscillators: part II-resonance capture. J. Appl. Mech. 68(1), 42-48 (2001)

6. Vakakis, A.F.: Shock isolation through the use of nonlinear energy sinks. J. Vib. Control. 9, 79-93 (2003)

7. Vakakis, A.F., Manevitch, L.I., Gendelman, O.V., Bergman, L.: Dynamics of linear discrete systems connected to local, essentially non-linear attachments. J. Sound Vib. 264, 559-577 (2003)

8. Gendelman, O.V.: Bifurcations of nonlinear normal modes of linear oscillator with strongly nonlinear damped attachment. Nonlinear Dyn. 37, 115-128 (2004)

9. Gendelman, O.V., Lamarque, C.-H.: Dynamics of linear oscillator coupled to strongly nonlinear attachment with multiple states of equilibrium. Chaos Soliton Frac. 24, 501-509 (2005)

10. Kerschen, G., Vakakis, A.F., Lee, Y.S., McFarland, D.M., Kowtko, J.J., Bergman, L.A.: Energy transfers in a system of two coupled oscillators with essential nonlinearity: 1:1 resonance manifold and transient bridging orbits. Nonlinear Dyn. 42, 283-303 (2005)

11. Gourdon, E., Lamarque, C.-H.: Energy pumping for a larger span of energy. J. Sound Vib. 285, 711-720 (2005)

12. Gendelman, O.V., Gourdon, E., Lamarque, C.-H.: Quasiperiodic energy pumping in coupled oscillators under periodic forcing. J. Sound Vib. 294, 651-662 (2006)

13. Kerschen, G., Lee, Y.S., Vakakis, A.F., McFarland, D.M., Bergman, L.A.: Irreversible passive energy transfer in coupled oscillators with essential nonlinearity. SIAM J. Appl. Math. 66, 648-679 (2006) 
14. Manevitch, L.I., Gourdon, E., Lamarque, C.-H.: Parameters optimization for energy pumping in strongly nonhomogeneous 2 dof system. Chaos Soliton Frac. 31, 900-911 (2007)

15. Manevitch, L.I., Musienko, A.I., Lamarque, C.-H.: New analytical approach to energy pumping problem in strongly nonhomogeneous 2dof systems. Meccanica 42, 77-83 (2007)

16. Lee, Y.S., Vakakis, A.F., Bergman, L.A., McFarland, D.M., Kerschen, G.: Suppression of aeroelastic instabilities by means of targeted energy transfers: part I, theory. AIAA J. 45, 693-711 (2007)

17. Panagopoulos, P.N., Gendelman, O.V., Vakakis, A.F.: Robustness of targeted energy transfer in coupled oscillators to changes of initial conditions. Nonlinear Dyn. 47, 377-387 (2007)

18. Lee, Y.S., Vakakis, A.F., Bergman, L.A., McFarland, D.M., Kerschen, G., Nucera, F., Tsakirtzis, S., Panagopoulos, P.N.: Passive nonlinear targeted energy transfer and its applications to vibration absorption: a review. Proc. Inst. Mech. Eng. K J. Multi-body Dyn. 222, 77-134 (2008)

19. Vakakis, A.F., Gendelman, O.V., Bergman, L.A., McFarland, D.M., Kerschen, G., Lee, Y.S.: Nonlinear Trageted Energy Transfer in Mechanical and Structural Systems I. Springer, Berlin (2008)

20. Vakakis, A.F., Gendelman, O.V., Bergman, L.A., McFarland, D.M., Kerschen, G., Lee, Y.S.: Nonlinear Trageted Energy Transfer in Mechanical and Structural Systems II. Springer, Berlin (2008)

21. Starosvetsky, Y., Gendelman, O.V.: Strongly modulated response in forced 2DOF oscillatory system with essential mass and potential asymmetry. Physica D. 237, 1719-1733 (2008)

22. Viguie, R., Peeters, M., Kerschen, G., Golinval, J.C.: Energy transfer and dissipation in a Duffing oscillator coupled to a nonlinear attachment. J. Comput. Nonlinear Dyn. 4, 041012 (2009)

23. Starosvetsky, Y., Gendelman, O.V.: Vibration absorption in systems with a nonlinear energy sink: nonlinear damping. J. Sound Vib. 324, 916-939 (2009)

24. Sapsis, T.P., Vakakis, A.F., Gendelman, O.V., Bergman, L.A., Kerschen, G., Quinn, D.D.: Efficiency of targeted energy transfers in coupled nonlinear oscillators associated with 1:1 resonance captures: part II, analytical study. J. Sound Vib. 325, 297-320 (2009)

25. Pham, T.T., Pernot, S., Lamarque, C.-H.: Competitive energy transfer between a two degreeof-freedom dynamic system and an absorber with essential nonlinearity. Nonlinear Dyn. 62, 573-592 (2010)

26. Gendelman, O.V., Vakakis, A.F., Bergman, L.A., McFarland, D.M.: Asymptotic analysis of passive nonlinear suppression of aeroelastic instabilities of a rigid wing in subsonic flow. SIAM J. Appl. Math. 70, 1655-1677 (2010)

27. Vaurigaud, B., Ture Savadkoohi, A., Lamarque, C.-H.: Targeted energy transfer with parallel nonlinear energy sinks part I: design theory and numerical results. Nonlinear Dyn. 66(4), 763-780 (2011)

28. Vaurigaud, B., Manevitch, L.I., Lamarque, C.-H.: Passive control of aeroelastic instability in a long span bridge model prone to coupled flutter using targeted energy transfer. J. Sound Vib. 330(11), 2580-2595 (2011)

29. Ture Savadkoohi, A., Manevitch, L.I., Lamarque, C.-H.: Analysis of the transient behavior in a two dof nonlinear system. Chaos Soliton Frac. 44(6), 450-463 (2011)

30. Starosvetsky, Y., Gendelman, O.V.: Response regimes in forced system with non-linear energy sink: quasi-periodic and random forcing. Nonlinear Dyn. 64, 77-195 (2011)

31. Gendelman, O.V., Sigalov, G., Manevitch, L.I., Mane, M., Vakakis, A.F., Bergman, L.A.: Dynamics of an eccentric rotational nonlinear energy sink. J. Appl. Mech. T. ASME J. 79, 011012 (2012)

32. Pham, T.T., Lamarque, C.-H., Ture Savadkoohi, A.: Multi-resonance capturing in a two-degreeof-freedom system under two different harmonic excitations. J. Vib. Control. 18(3), 451-466 (2012)

33. Luongo, A., Zulli, D.: Dynamic analysis of externally excited NES-controlled systems via a mixed multiple scale/harmonic balance algorithm. Nonlinear Dyn. 70, 2049-2061 (2012)

34. Luongo, A., Zulli, D.: Aeroelastic instability analysis of NES-controlled systems via a mixed multiple scale/harmonic balance method. J. Vib. Control. 20, 1985-1998 (2014) 
35. Wierschem, N.E., Quinn, D.D., Hubbard, S.A., Al-Shudeifatd, M.A., McFarlandc, D.M., Luo, J., Fahnestock, L.A., Spencer Jr, B.F., Vakakis, A.F., Bergman, L.A.: Passive damping enhancement of a two-degree-of-freedom system through a strongly nonlinear two-degree-of-freedom attachment. J. Sound Vib. 331, 5393-5407 (2012)

36. McFarland, D.M., Kerschen, G., Kowtko, J.J., Lee, Y.S., Bergman, L.A., Vakakis, A.F.: Experimental investigation of targeted energy transfers in strongly and nonlinearly coupled oscillators. J. Acoust. Soc. Am. 118, 791-799 (2005)

37. Kerschen, G., Kowtko, J.J., McFarland, D.M., Bergman, L.A., Vakakis, A.F.: Theoretical and experimental study of multimodal targeted energy transfer in a system of coupled oscillators. Nonlinear Dyn. 47, 285-309 (2007)

38. Kerschen, G., Kowtko, J.J., McFarland, D.M., Lee, Y.S., Bergman, L.A., Vakakis, A.F.: Experimental demonstration of transient resonance capture in a system of two coupled oscillators with essential stiffness nonlinearity. J. Sound Vib. 299, 822-838 (2007)

39. Gourdon, E., Alexander, N.A., Taylor, C.A., Lamarque, C.-H., Pernot, S.: Nonlinear energy pumping under transient forcing with strongly nonlinear coupling: theoretical and experimental results. J. Sound Vib. 300, 522-551 (2007)

40. Lee, Y.S., Kerschen, G., McFarland, D.M., Hill, W.J., Nichkawde, C., Strganac, T.W., Bergman, L.A., Vakakis, A.F.: Suppressing aeroelastic instability using broadband passive targeted energy transfers, part 2: experiments. AIAA J. 45, 2391-2400 (2007)

41. Vaurigaud, B., Ture Savadkoohi, A., Lamarque, C.-H.: Efficient targeted energy transfer with parallel nonlinear energy sinks: theory and experiments. J. Comput. Nonlinear Dyn. 6(4), 041005 (2011)

42. True Savadkoohi, A., Vaurigaud, B., Lamarque, C.-H., Pernot, S.: Targeted energy transfer with parallel nonlinear energy sinks, part II: theory and experiments. Nonlinear Dyn. 67, 3746 (2012)

43. Wierschem, N., Luo, J., AL-Shudeifat, M., Hubbard, S., Ott, R., Fahnestock, L., Quinn, D., McFarland, D., Spencer Jr, B., Vakakis, A., Bergman, L.: Experimental testing and numerical simulation of a six-story structure incorporating two-degree-of-freedom nonlinear energy sink. J. Struct. Eng. 140(6), 04014027 (2014)

44. Gendelman, O.V.: Analytic treatment of a system with a vibro-impact nonlinear energy sink. J. Sound Vib. 331, 4599-4608 (2012)

45. Gendelman, O.V.: Targeted energy transfer in systems with non-polynomial nonlinearity. J. Sound Vib. 315, 732-745 (2008)

46. Lamarque, C.-H., Gendelman, O.V., Ture Savadkoohi, A., Etcheverria, E.: Targeted energy transfer in mechanical systems by means of non-smooth nonlinear energy sink. Acta Mech. 221, 175-200 (2011)

47. Nucera, F., Vakakis, A.F., McFarland, D.M., Bergman, L.A., Kerschen, G.: Targeted energy transfers in vibro-impact oscillators for seismic mitigation. Nonlinear Dyn. 50, 651-677 (2007)

48. True Savadkoohi, A., Lamarque, C.-H., Dimitrijevic, Z.: Vibratory energy exchange between a linear and a non-smooth system in the presence of the gravity. Nonlinear Dyn. 70, 1473-1483 (2012)

49. Ture Savadkoohi, A., Lamarque, C.-H.: Vibratory energy localization by non-smooth energy sink with time-varying mass. Appl. Non-Linear Dyn. Syst. Springer Proc. Math. Stat. 93, 429-442 (2014)

50. Lamarque, C.-H., Ture Savadkoohi, A., Dimitrijevic, Z.: Dynamics of a linear system with time-dependant mass and a coupled light mass with non-smooth potential. Meccanica 49, $135-145$ (2014)

51. Lamarque, C.-H., Ture Savadkoohi, A., Etcheverria, E., Dimitrijevic, Z.: Multi-scales dynamics of two coupled non-smooth systems. Int. J. Bifurcat. Chaos. 22(1250295), 1-18 (2012)

52. True Savadkoohi, A., Lamarque, C.-H.: Dynamics of coupled Dahl type and non-smooth systems at different scales of time. Int. J. Bifurcat. Chaos 23(1350114), 1-14 (2013)

53. Lamarque, C.-H., Ture Savadkoohi, A.: Dynamical behavior of a Bouc-Wen type oscillator coupled to a nonlinear energy sink. Meccanica 49, 1917-1928 (2014) 
54. Bastien, J., Bernardin, F., Lamarque, C.-H.: Non Smooth Deterministic or Stochastic Discrete Dynamical Systems: Applications to Models with Friction or Impact, p. 515. Wiley, USA (2013)

55. Weiss, M., Ture Savadkoohi, A., Gendelman, O.V., Lamarque, C.-H.: Dynamical behavior of a mechanical system including Saint-Venant component coupled to a nonlinear energy sink. Int. J. Non-Linear Mech. 63, 10-18 (2014)

56. Lamarque, C.-H.: Ture Savadkoohi, A.: Targeted energy transfer between a system with a set of Saint-Venant elements and a nonlinear energy sink. Continuum Mech. Thermodyn. (2014). doi:10.1007/s00161-014-0354-9

57. Manevitch, L.I.: The description of localized normal modes in a chain of nonlinear coupled oscillators using complex variables. Nonlinear Dyn. 25, 95-109 (2001)

58. Nayfeh, A.H., Mook, D.T.: Nonlinear Oscillations. Wiley, New York (1979)

59. Schmidt, F., Lamarque, C.-H.: Energy pumping for mechanical systems involving non-smooth Saint-Venant terms. Int. J. Nonlinear Mech. 45, 866-875 (2010)

60. Wang, D.H., Liao, W.H.: Magnetorheological fluid dampers: a review of parametric modelling. Smart Mater. Struct. 20, 023001 (34pp) (2011) 